\title{
Wireless Optical Communications Through the Turbulent Atmosphere: A Review
}

\author{
Ricardo Barrios and Federico Dios \\ Department of Signal Theory and Communications, Technical University of Catalonia \\ Spain
}

\section{Introduction}

In the past decades a renewed interest has been seen around optical wireless communications, commonly known as free-space optics (FSO), because of the ever growing demand for high-data-rate data transmission as to a large extent current applications, such as the high-definition (HD) contents and cloud computing, require great amount of data to be transmitted, hence, demanding more transmission bandwidth. Nowadays, the last mile problem continuous to be the bottle neck in the global communication network. While the fiber-optic infrastructure, commonly called network backbone, is capable of coping with current demand, the end user accesses the network data stream through copper based connection and radio-frequency (RF) wireless services, that are inherently slower technologies. As the number of user increases, the radio-frequency spectrum is getting so crowded that there is virtually no room for new wireless services within the RF band, with the added inconvenient of limited bandwidth restriction when using a RF band and the license fees that have to be paid in order to use such a band. Regarding cooper-based technologies and the lower-speed connections, compared with the backbone, that are offered such as DSI (digital line subscriber), cable modems, or T1's (transmission system 1), they are alternatives that makes the service provider to incur in extra installation costs for deploying the wired network through the city.

When a fiber-optic link is neither practical nor feasible, under the above scenario, wireless optical communications (WOC) becomes a real alternative, since it allows to transfer data with high-bandwidth requirements with the additional advantages of wireless systems (Arimoto, 2010; Ciaramella et al., 2009; Sova et al., 2006). Moreover, a wireless optical communication system offers, when compared with RF technology, an intrinsic narrower beam; less power, mass and volume requirements, and the advantage of no regulatory policies for using optical frequencies and bandwidth.

On the other hand, satellite communication systems is a field where FSO is becoming more attractive thanks to the advantages mentioned above, and the additional fact that for satellite-satellite links there is no beam degradation due to the absence of atmosphere. Nevertheless, the pointing system complexity is increased as the order of the optical beam divergence is hundreds of $\mu \mathrm{rad}$, whereas for an RF beam is in the order of tens to hundreds of mrad. The Semi-Conductor Inter Satellite Link EXperiment (SILEX) was the first European project to conduct a successful demo with the transmission of data through 
an optical link, between the SPOT-4 and Artemis satellites achieving 50Mbps of transfer rate (Fletcher et al., 1991). There has also been experiments for ground-satellite optical links such as the Ground/Orbiter Lasercomm Demonstrator (GOLD) (Jeganathan et al., 1997); and for air-satellite link with the Airbone Atmospheric Laser Link (LOLA, for its French initials), which used of the Artemis optical payload and an airborne optical transceiver flying at $9000 \mathrm{~m}$ (Cazaubiel et al., 2006).

The major drawback for deploying wireless links based on FSO technology, where lasers are used as sources, is the perturbation of the optical wave as it propagates through the turbulent atmosphere. Moreover, fog, rain, snow, haze, and generally any floating particle can cause extinction of the signal-carrying laser beam intensity. In a worst case scenario the intensity attenuation can be strong enough to cause link outages, leading to a high bit error-rate that inevitably decrease the overall system performance and limits the maximum length for the optical link.

The turbulent atmosphere produces many effects, of which the most noticeable is the random fluctuations of the traveling wave irradiance, phenomenon known as scintillation. Additionally, there are other effects that perturb the traveling wavefront such as beam wander, that is a continuous random movement of the beam centroid over the receiving aperture; angle-of-arrival fluctuations, which are associated with the dancing of the focused spot on the photodetector surface; and beam spreading that is the spreading beyond the pure diffraction limit of the beam radius.

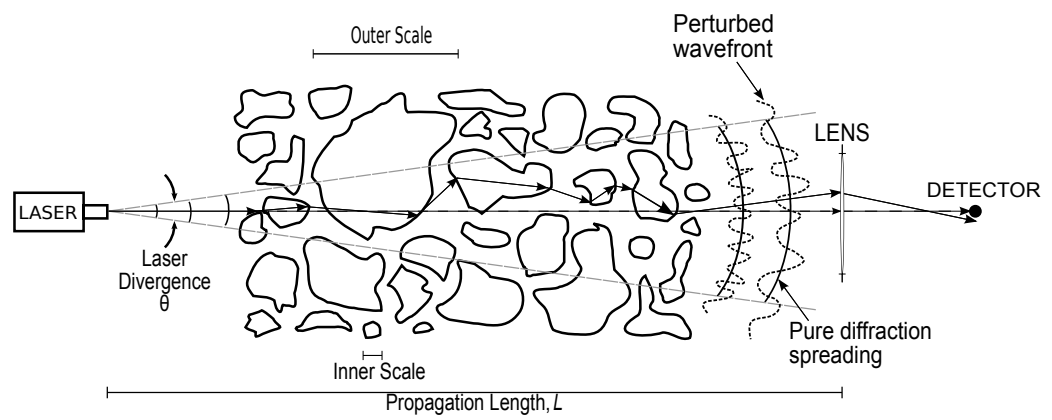

Fig. 1. Laser beam propagation through the turbulent atmosphere.

A compound of various perturbations suffered by an optical traveling wavefront is shown in Fig. 1. Here, the minor spatial scale distortions in the wavefronts provoke a random pattern, both in time and space, of self-interference of the beam at the receiver plane. As a consequence rapid variations of the received power appear, which is the most noticeable effect of the atmospheric turbulence over the optical link. The rays (solid arrows) leaving the laser source are deflected as they travel through the largest air pockets, whose size defines the turbulence outer scale, arriving off-axis instead of what is expected without turbulence, represented with the straight dashed arrow starting at laser and finishing at the receptor surface in Fig. 1. Additionally, the turbulent atmosphere induces a spreading of the beam that is the broadening of the beam size beyond of that expected due to pure diffraction, for the case of a laser beam. It is customary to refer as refractive effects to those caused by the outer scale size of turbulence, whereas, the inner scale sizes produces the diffractive effects. As the rays may also be interpreted as the wave vector for the traveling wavefront, the variations 
in the angle respect the optical axis at the receiver represent the concept of angle-of-arrival fluctuations. Furthermore, this bouncing of the optical wavefront as it propagates through the atmosphere is also responsible for the beam wander effect as the centroid of the laser beam is displaced randomly at the receiver plane.

This chapter is organized as follows: In Section 2 the most widely spread power spectrum models to characterize the turbulent atmosphere are addressed. Secondly, in Section 3, a short yet complete review of the propagation of optical electromagnetic waves in turbulent media is presented, followed by a brief introduction to the beam split-step method for the simulation of traveling optical beams in Section 4. Finally, WOC systems are addressed from a communication theory approach where general system characterization and performance evaluation are made in Section 5 and Section 6,respectively.

\section{Atmospheric turbulence}

All the models used to describe the effects of the atmosphere on a optical traveling wave are based on the study of turbulence, which involves fluctuations in the velocity field of a viscous fluid (Andrews \& Philips, 2005). These variations in the fluid-the air for the atmosphere case-are firstly due to temperature differences between the surface of the Earth and the atmosphere, then, to the differences in temperature and pressure within the atmospheric layers themselves, thus, producing pockets of air, also known as eddies, that cause the atmospheric turbulence.

The different eddy sizes, called the inertial range, responsible for the transfer of kinetic energy within the fluid go from the outer scale $L_{0}$ to the inner scale $l_{0}$ of turbulence, where typical values of $L_{0}$ are between 10 and $20 \mathrm{~m}$, while $l_{0}$ is usually around $1-2 \mathrm{~mm}$. Such conditions comprise a continuum where wind energy is injected in the macroscale $L_{0}$, transfer through the inertial range and finally dissipated in the microscale $l_{0}$. This energy transfer causes unstable air masses, with temperature gradients, giving rise to local changes in the atmospheric refractive-index and thus creating what is called optical turbulence as an optical wave propagates. Treating the atmospheric turbulence as a consequence of the fluctuations in refractive-index instead of temperature is the natural way to address wave propagation for optical frequencies. Following this reasoning is a good approach to define a power spectral density for refractive-index fluctuations as a mean to express the atmospheric turbulence.

The variations of the atmospheric refractive-index $n$, which can be considered as locally homogeneous, can be mathematically expressed by

$$
n(\vec{r}, t)=n_{0}+n_{1}(\vec{r}, t),
$$

where $n_{0}$ is the mean value of the index of refraction; $n_{1}(\vec{r}, t)$ is a random variable with zero mean, representing the changes caused by the atmospheric turbulence, and $t$ indicates the temporal dependence. Nevertheless, under the Taylor frozen turbulence hypothesis, the turbulence is regarded as stationary as the optical wave propagates, hence, the time dependence is traditionally dropped in Eq. (1).

The statistical characterization of a locally homogeneous random field is usually done by its structure function, denoted by

$$
D_{n}\left(\overrightarrow{r_{1}}, \overrightarrow{r_{2}}\right)=\left\langle\left[n\left(\overrightarrow{r_{1}}\right)-n\left(\overrightarrow{r_{2}}\right)\right]^{2}\right\rangle,
$$

where there is no time dependence in the index of refraction. 


\subsection{Refractive-index structure parameter}

The atmospheric turbulence can be defined by the strength of the fluctuations in the refractive-index, represented with the refractive-index structure parameter $C_{n}^{2}$ in units of $\mathrm{m}^{-2 / 3}$ - which has a direct relation with the structure function mentioned above. Along the optical propagation distance the value of $C_{n}^{2}$ have small variations for horizontal paths, while for slant and vertical paths these variations become significant. It's very common to assume a constant value for horizontal links, and to measure the path averaged value of $C_{n}^{2}$ from methods that rely on the atmospheric data in situ (Andreas, 1988; Doss-Hammel et al., 2004; Lawrence et al., 1970; Sadot \& Kopeika, 1992), or others that extract the $C_{n}^{2}$ value from experimental scintillation data (Fried, 1967; Wang et al., 1978).

On the other hand, when a vertical path is considered, the behavior of $C_{n}^{2}$ is conditioned by temperature changes along the different layers within the Earth's atmosphere, hence, the refractive-index structure parameter becomes a function of the altitude above ground.

\begin{tabular}{|c|c|}
\hline Model & Expression \\
\hline SLC-Day & $C_{n}^{2}(h)=\left\{\begin{array}{lc}1.700 \cdot 10^{-14} & 0 \mathrm{~m}<h<19 \mathrm{~m} \\
4.008 \cdot 10^{-13} h^{-1.054} & 19 \mathrm{~m}<h<230 \mathrm{~m} \\
1.300 \cdot 10^{-15} & 230 \mathrm{~m}<h<850 \mathrm{~m} \\
6.352 \cdot 10^{-7} h^{-2.966} & 850 \mathrm{~m}<h<7000 \mathrm{~m} \\
6.209 \cdot 10^{-16} h^{-0.6229} & 7000 \mathrm{~m}<h<20000 \mathrm{~m}\end{array}\right.$ \\
\hline Hufnagel-Valley Day & $C_{n}^{2}(h)=A e^{\frac{-h}{100}}+5.94 \cdot 10^{-53}\left(\frac{v}{27}\right)^{2} h^{10} e^{\frac{-h}{1000}}+2.7 \cdot 10^{-16} e^{\frac{-h}{1500}}$ \\
\hline Hufnagel-Valley Night & $C_{n}^{2}(h)=1.9 \cdot 10^{-15} e^{\frac{-h}{100}}+8.16 \cdot 10^{-54} h^{10} e^{\frac{-h}{1000}}+3.02 \cdot 10^{-17} e^{\frac{-h}{1500}}$ \\
\hline Greenwood & $C_{n}^{2}(h)=\left[2.2 \cdot 10^{-13}(h+10)^{-1.3}+4.3 \cdot 10^{-17}\right] e^{-h / 1500}$ \\
\hline
\end{tabular}

Table 1. Refractive-index structure parameter models as a function of the altitude $h$ above ground. For the HV model, $A=C_{n}^{2}(0)$ is the refractive-index structure parameter at ground level, and $v$ is the root-mean-square wind speed.

Many authors have tried to predict the behavior of the refractive-index structure parameter, and various models have been proposed. However, it should be noted that most of these models are based on fittings from experiments conducted in specific places, which makes difficult their generalization. Table 1 presents a list of different $C_{n}^{2}$ models, namely, the Submarine Laser Communication (SLC) Day model and the Hufnagel-Valley, best suited for inland day-time conditions, the HV-Night for night-time conditions, and the Greenwood model adapted for astronomical tasks from mountaintop locations. A comparative of all four refractive-index structure parameter models is shown below in Fig. 2, where it is readily seen that day-time models predict higher values of $C_{n}^{2}$ than night-time models, as expected.

Sadot \& Kopeika (1992) have developed an empirical model for estimating the refractive-index structure parameter from macroscale meteorological measurements in situ. The value of $C_{n}^{2}$ depends strongly on the hour of the day. It has a peak value at midday and local minima at sunrise and sunset. Provided that the time elapsed between the sunrise and sunset is different according to seasonal variations, the concept of temporal hour $\left(t_{h}\right)$ has been introduced. The duration of a temporal hour is $1 / 12^{\text {th }}$ of the time between sunrise and 


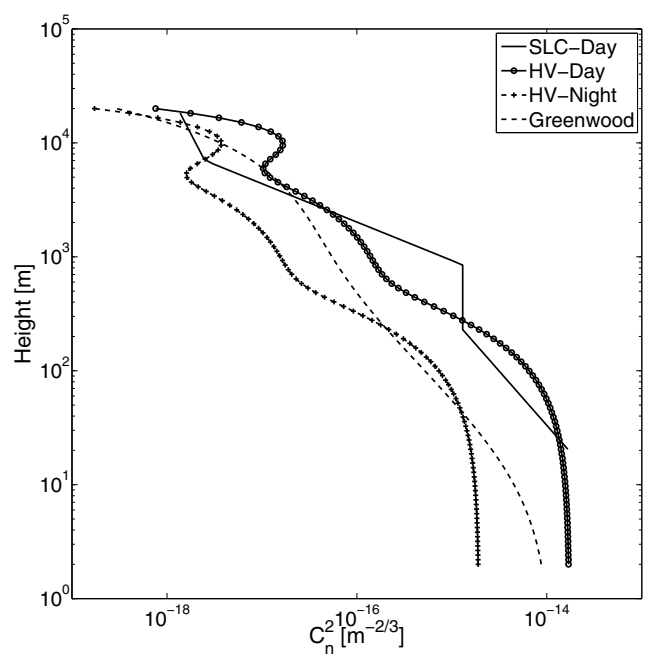

Fig. 2. Refractive-index structure parameter altitude profile of different models. For HV-day model $A=1.7 \cdot 10^{-14} \mathrm{~m}^{-2 / 3}$ and $v=21 \mathrm{~m} / \mathrm{s}$.

sunset. In summer it is more than $60 \mathrm{~min}$ and in winter is smaller, therefore, it can be seen as a solar hour. The current $t_{h}$ is obtained by subtracting the sunrise time from the local time, and dividing by the value of one $t_{h}$. Thus, in any day of the year $t_{h}=00: 00$ at sunrise, $t_{h}=06: 00$ at noon, and $t_{h}=12: 00$ at sunset. It should be noted that temporal hours are allowed to have negative time hours.

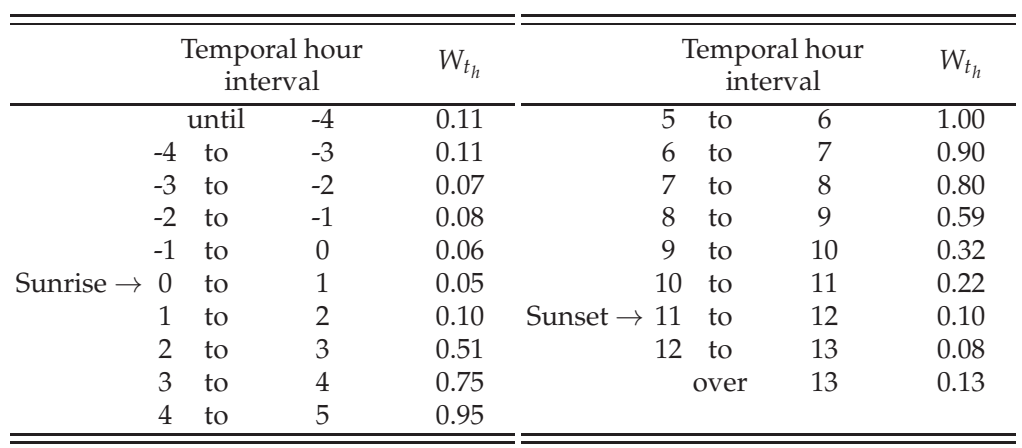

Table 2. Weight $W$ parameter as a function of the corresponding temporal hour.

The expression obtained that describes $C_{n}^{2}$ is based on a polynomial regression model according to

$$
\begin{aligned}
C_{n}^{2}= & 3.8 \times 10^{-14} W_{t_{h}}+2 \times 10^{-15} \mathrm{~T}-2.8 \times 10^{-15} \mathrm{RH}+2.9 \times 10^{-17} \mathrm{RH}^{2}-1.1 \times 10^{-19} \mathrm{RH}^{3} \\
& -2.5 \times 10^{-15} \mathrm{WS}+1.2 \times 10^{-15} \mathrm{WS}^{2}-8.5 \times 10^{-17} \mathrm{WS}^{3}-5.3 \times 10^{-13},
\end{aligned}
$$

where $W_{t_{h}}$ denotes a temporal-hour weight (see Table 2), $T$ is the temperature in Kelvins, $R H$ is the relative humidity (\%), and WS is the wind speed in $\mathrm{m} \mathrm{s}^{-1}$. 
An improved version of this model is also presented in Sadot \& Kopeika (1992), with the introduction of the effects of solar radiation and aerosol loading in the atmosphere, as follows

$$
\begin{aligned}
C_{n}^{2}= & 5.9 \times 10^{-15} W_{t_{h}}+1.6 \times 10^{-15} \mathrm{~T}-3.7 \times 10^{-15} \mathrm{RH}+6.7 \times 10^{-17} \mathrm{RH}^{2} \\
& -3.9 \times 10^{-19} \mathrm{RH}^{3}-3.7 \times 10^{-15} \mathrm{WS}+1.3 \times 10^{-15} \mathrm{WS}^{2}-8.2 \times 10^{-17} \mathrm{WS}^{3} \\
& +2.8 \times 10^{-14} \mathrm{SF}-1.8 \times 10^{-14} \mathrm{TCSA}+1.4 \times 10^{-14} \mathrm{TCSA}^{2}-3.9 \times 10^{-13},
\end{aligned}
$$

where SF is the solar flux in units of $\mathrm{kW} \mathrm{m}^{-2}$, and TCSA is the total cross-sectional area of the aerosol particles and its expression can be found in Yitzhaky et al. (1997)

$$
\begin{aligned}
\text { TCS } A= & 9.96 \times 10^{-4} \mathrm{RH}-2.75 \times 10^{-5} \mathrm{RH}^{2}+4.86 \times 10^{-7} \mathrm{RH}^{3}-4.48 \times 10^{-9} \mathrm{RH}^{4} \\
& +1.66 \times 10^{-11} \mathrm{RH}^{5}-6.26 \times 10^{-3} \ln \mathrm{RH}-1.37 \times 10^{-5} \mathrm{SF}^{4}+7.30 \times 10^{-3} .
\end{aligned}
$$

A 24-hour data set of macroscale meteorological measurements taken at the Campus Nord in the Technical University of Catalonia in Barcelona, Spain, collected on the $14^{\text {th }}$ of November of 2009 was used to generate the plot, shown in Fig. 3, of the estimated refractive-index structure parameter $C_{n}^{2}$ using Eq. (3) and Eq. (4) labeled as Model 1 and Model 2, respectively.

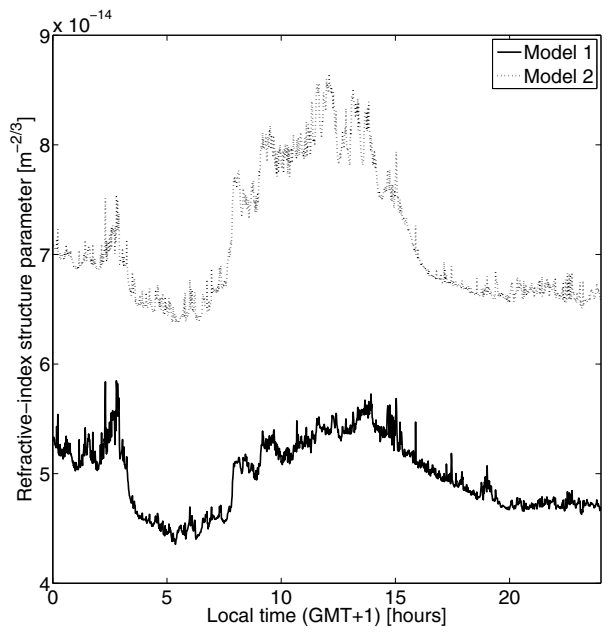

Fig. 3. Refractive-index structure parameter predicted from macroscale meteorological and aerosols data collected in an autumn day in Barcelona, Spain.

\subsection{Atmospheric power spectrum models}

The first studies on the atmospheric turbulence effects on propagating light waves were conducted by Tatarskii (1971) using the Rytov method and considering, as still does nowadays, the Kolmogorov turbulence spectrum (Kolmogorov, 1941) that suggest that the inertial range has a degree of statistical consistency, where points in the atmosphere separated certain scale size exhibit statistical homogeneity and isotropy. The use of these characteristics, along with additional simplifications and assumptions, were essential to develop tractable expressions for a fundamentally nonlinear phenomenon, as the atmospheric turbulence. 
Kolmogorov was the first to derive an expression, which led to the spectrum model

$$
\Phi_{n}(\kappa)=0.033 C_{n}^{2} \kappa^{-11 / 3},
$$

where $\kappa$ is the scalar spatial frequency (in $\operatorname{rad~m}^{-1}$ ).

Although Eq. (6) is only valid over th inertial subrange, $1 / L_{0} \ll \kappa \ll 1 / l_{0}$, it is often assumed that the outer scale is infinite and the inner scale is negligibly small in order to make use of it for all spatial frequencies. However, in practice, making this assumption can lead to untrustworthy results when using the Kolmogorov spectrum for spatial frequencies out of the actual inertial range.

To overcome the singularities appearing in Eq. (6) other spectrum models have been proposed. Tatarskii suggested to include the inner scale effects with a Gaussian function, defining a new power spectral density for refractive-index fluctuations in the form

$$
\Phi_{n}(\kappa)=0.033 C_{n}^{2} \kappa^{-11 / 3} \exp \left(-\frac{\kappa^{2}}{\kappa_{m}^{2}}\right), \quad \kappa \gg 1 / L_{0} ; \quad \kappa_{m}=5.92 / l_{0} .
$$

The Tatarskii spectrum still presents a mathematical singularity at $\kappa=0$ in the limiting case $L_{0} \rightarrow \infty$. A further improvement of the Tatarskii and Kolmogorov spectrum, valid for all spatial frequencies, called the von Kármán spectrum is given by the expression

$$
\Phi_{n}(\kappa)=0.033 C_{n}^{2} \frac{\exp \left(-\kappa^{2} / \kappa_{m}^{2}\right)}{\left(\kappa^{2}+\kappa_{0}^{2}\right)^{11 / 3}}, \quad 0 \leq \kappa<\infty ; \quad \kappa_{m}=5.92 / l_{0},
$$

where $\kappa_{0}=2 \pi / L_{0}$.

It should be noted that both Eq. (7) and Eq. (8) reduce to the Kolmogorov power spectrum, when evaluated in the inertial range $\kappa_{0} \ll \kappa \ll \kappa_{m}$.

The spatial power spectral density of refractive-index fluctuations, as being derived from a locally homogeneous random field, is described by its structure function defined by

$$
D_{n}(\vec{r})=8 \pi \int_{0}^{\infty} \kappa^{2} \Phi_{n}(\vec{\kappa})\left(1-\frac{\sin \vec{\kappa} \cdot \vec{r}}{\vec{\kappa} \cdot \vec{r}}\right) d \vec{\kappa}
$$

where $\Phi_{n}(\vec{\kappa})$ is the power spectrum model of interest.

\section{Propagation theory}

An optical wave propagating through the atmosphere will be altered by refractive-index inhomogeneities that form turbulent eddies of different sizes, where energy is injected in the macroscale $L_{0}$ and transfered through ever smaller eddies and finally is dissipated at the microscale $l_{0}$. This energy transfer causes unstable air masses, with temperature gradients, giving rise to local changes in the atmospheric refractive-index and thus inducing perturbations as the optical wave propagates.

The random variations on the amplitude and phase of the traveling wave can be addressed theoretical, by solving the wave equation for the electric field and its respective statistical moments. For a propagating electromagnetic wave the electric field is derived from the stochastic Helmholtz equation

$$
\nabla^{2} \vec{E}+k^{2} n^{2}(\vec{r}) \vec{E}=0,
$$


where $k=2 \pi / \lambda$ is the wavenumber, $\vec{r}$ is a point in the space and $n(\vec{r})$ is given by Eq. (1).

Traditionally, the actual equation to be solved is the scalar stochastic Helmholtz equation

$$
\nabla^{2} U+k^{2} n^{2}(\vec{r}) U=0,
$$

which corresponds to one of three components of the electric field.

To solve Eq. (11) the Born and Rytov approximations have traditionally been used. Additionally, several assumptions are made, namely, backscattering and depolarization effects are neglected, the refractive-index is assumed uncorrelated in the direction of propagation, and the paraxial approximation can be used.

\subsection{Born approximation}

In the Born approximation the solution of Eq. (11) is assumed to be a sum of terms of the form

$$
U(\vec{r})=U_{0}(\vec{r})+U_{1}(\vec{r})+U_{2}(\vec{r})+\cdots,
$$

where $U_{0}(\vec{r})$ represents the unperturbed field-i.e. an optical wave traveling through free-space. While $U_{1}(\vec{r})$ and $U_{2}(\vec{r})$ denote first-order, second-order, and so on, perturbations caused by inhomogeneities due to the random term $n_{1}(\vec{r})$ in Eq. (1).

Next, by using the fact that in Eq. (1) $n_{0} \cong 1$ and $\left|n_{1}(\vec{r})\right| \ll 1$, Eq. (11) reduces to

$$
\nabla^{2} U(\vec{r})+k^{2}\left[1+2 n_{1}(\vec{r})\right] U(\vec{r})=0,
$$

Finally, substituting Eq. (12) into Eq. (13) yields to (Andrews \& Philips, 2005)

$$
\begin{aligned}
& \nabla^{2} U_{0}+k^{2} U_{0}=0, \\
& \nabla^{2} U_{1}+k^{2} U_{1}=-2 k^{2} n_{1}(\vec{r}) U_{0}(\vec{r}), \\
& \nabla^{2} U_{2}+k^{2} U_{2}=-2 k^{2} n_{1}(\vec{r}) U_{1}(\vec{r}),
\end{aligned}
$$

and so on for higher order perturbations terms.

Solving Eq. (14) gives the unperturbed propagated optical field, whereas solving Eq. (15) and Eq. (16) give the two lower-order perturbed fields. Next, a brief explanation on how to solve this system of equations is given below.

\subsubsection{Unperturbed field}

Let us refer to Fig. 1 and consider a Gaussian beam wave propagating in the $z$ direction, where the input plane $z=0$ of the system is located at the output of the laser, and the output plane is located at the receiver lens position. The initial field can be described by (Ishimaru, 1969)

$$
U_{0}(r, 0)=A \exp \left(-\frac{r^{2}}{W_{0}^{2}}\right) \exp \left(-i \frac{k r^{2}}{2 F_{0}}\right),
$$

where $r$ is the distance from the beam center, and $W_{0}$ and $F_{0}$ are the beam radius and phase front radius at the transmitter plane, respectively. ${ }^{1}$

\footnotetext{
${ }^{1}$ The notation used in this section is taken from Andrews \& Philips (2005). Special care have to be taken with this notation, where $W_{0}$ is specifically referring to the beam radius at the output of the light source, and it should not be confused with the actual beam waist of a Gaussian beam $W_{B}$.
} 
Furthermore, the Gaussian beam can be characterized by the input parameters

$$
\begin{aligned}
& \Theta_{0}=1-\frac{z}{F_{0}}, \\
& \Lambda_{0}=\frac{2 z}{k W_{0}^{2}},
\end{aligned}
$$

and by the output parameter in the receiver plane at $z=L$

$$
\begin{aligned}
& \Theta=1+\frac{L}{F}=\frac{\Theta_{0}}{\Theta_{0}^{2}+\Lambda_{0}^{2}}, \\
& \Lambda=\frac{2 L}{k W^{2}}=\frac{\Lambda_{0}}{\Theta_{0}^{2}+\Lambda_{0}^{2}},
\end{aligned}
$$

where $W$ and $F$ are the beam radius and phase front radius at the receiver plane, respectively. The set of parameters defining a Gaussian beam presented above correspond to the notation used in Andrews \& Philips (2005). Nevertheless, other ways of characterizing a Gaussian beam can be utilized, such that used in Ricklin et al. (2006).

The solution of Eq. (11) for Gaussian beam wave propagating a distance $z$ in free-space is given by

$$
U_{0}(r, z)=\frac{1}{\sqrt{\Theta_{0}^{2}+\Lambda_{0}^{2}}} \exp \left(-\frac{r^{2}}{W^{2}}\right) \exp \left[i\left(k z-\varphi-\frac{k r^{2}}{2 F}\right)\right],
$$

where $\Theta_{0}$ and $\Lambda_{0}$ are non-dimensional parameters defined above, and $\varphi, W$, and $F$ are the longitudinal phase shift, beam radius, and radius of curvature after propagating a distance $z$. These quantities are defined by

$$
\begin{aligned}
\varphi & =\tan ^{-1} \frac{\Lambda_{0}}{\Theta_{0}}, \\
W & =W_{0} \sqrt{\Theta_{0}^{2}+\Lambda_{0}^{2}}, \\
F & =\frac{k W_{0}^{2}}{2}\left[\frac{\Lambda_{0}\left(\Theta_{0}+\Lambda_{0}\right)}{\Theta_{0}\left(1-\Theta_{0}\right)-\Lambda_{0}}\right] .
\end{aligned}
$$

\subsubsection{Perturbations terms}

For an optical wave propagating a distance $L$ in the $z$ direction, the first-order perturbation term of the output field is given by

$$
U_{1}(\vec{r})=2 k^{2} \iiint_{V} G(\vec{r}, \vec{s}) n_{1}(\vec{s}) U_{0}(\vec{s}) d \vec{s},
$$

where $U_{0}(\vec{s})$ and $G(\vec{r}, \vec{s})$ are the unperturbed field (see Eq. (22)) and the free-space Green's function (Yura et al., 1983), respectively. Moreover, by applying the paraxial approximation the first Born approximation reduces to

$$
U_{1}(\vec{r}, L)=\frac{k^{2}}{2 \pi} \int_{0}^{L} d z \iint_{\infty}^{\infty} d^{2} s \exp \left[i k(L-z)+\frac{i k|\vec{s}-\vec{r}|^{2}}{2(L-z)}\right] U_{0}(\vec{s}, z) \frac{n_{1}(\vec{s}, z)}{L-z} .
$$


When solving for higher-order perturbation terms in the Born approximation, the following recurrent formula can be used

$$
U_{m}(\vec{r}, L)=\frac{k^{2}}{2 \pi} \int_{0}^{L} d z \iint_{\infty}^{\infty} d^{2} s \exp \left[i k(L-z)+\frac{i k|\vec{s}-\vec{r}|^{2}}{2(L-z)}\right] U_{m-1}(\vec{s}, z) \frac{n_{1}(\vec{s}, z)}{L-z},
$$

where $m$ indicates the order of the perturbation term to be calculated.

\subsection{Rytov approximation}

The Rytov approximation assume a solution for Eq. (11) formed by the unperturbed field $U_{0}(\vec{r})$ modified by complex phase perturbation terms, expressed as

$$
U(\vec{r})=U_{0}(\vec{r}) \exp \left[\psi_{1}(\vec{r})+\psi_{2}(\vec{r})+\cdots\right],
$$

where $\psi_{1}(\vec{r})$ and $\psi_{2}(\vec{r})$ are first- and second-order phase perturbations terms, respectively. These perturbations are defined by (Yura et al., 1983)

$$
\begin{aligned}
& \psi_{1}(\vec{r})=\Phi_{1}(\vec{r}), \\
& \psi_{2}(\vec{r})=\Phi_{2}(\vec{r})-\frac{1}{2} \Phi_{1}^{2}(\vec{r}),
\end{aligned}
$$

where the new function $\Phi_{m}(\vec{r})$ appearing in the system of equations in Eq. (30) are directly related with the Born perturbation terms in the form

$$
\Phi_{m}(\vec{r})=\frac{U_{m}(\vec{r})}{U_{0}(\vec{r})}
$$

Historically, the Born approximation was first introduced but its results were limited to conditions of extremely weak scintillation. Afterwards, the second-order Rytov approximation won more acceptance thanks to the good agreement with scintillation data in the weak fluctuation regime.

\subsection{Statistical moments}

The first relevant statistical moment for a traveling optical field is the second-order moment, also known as the mutual coherence function (MCF), which is defined as the ensemble average over two points of the field, taken in a plane perpendicular to the propagation direction at a distance $L$ from the source, as follows

$$
\Gamma_{2}\left(\overrightarrow{r_{1}}, \overrightarrow{r_{2}}, L\right)=\left\langle U\left(\overrightarrow{r_{1}}, L\right) U^{*}\left(\overrightarrow{r_{2}}, L\right)\right\rangle,
$$

where $U(\vec{r})$ is the Rytov approximation solution for Eq. (11), and the brackets $\langle\cdot\rangle$ denote an ensemble average. Vectors $\overrightarrow{r_{1}}$ and $\overrightarrow{r_{2}}$ are transversal vectors without $z$ component, which is chosen as the propagation direction.

Solving Eq. (32)

$$
\Gamma_{2}\left(\overrightarrow{r_{1}}, \overrightarrow{r_{2}}, L\right)=\Gamma_{2}^{0}\left(\overrightarrow{r_{1}}, \overrightarrow{r_{2}}, L\right) \exp \left[\sigma_{r}^{2}\left(\overrightarrow{r_{1}}, L\right)+\sigma_{r}^{2}\left(\overrightarrow{r_{2}}, L\right)-T\right] \exp \left[\frac{-1}{2} \Delta(\vec{r}, \vec{r}, L)\right],
$$


where

$$
\begin{aligned}
\Gamma_{2}^{0}\left(\overrightarrow{r_{1}}, \overrightarrow{r_{2}}, L\right) & =U_{0}\left(\overrightarrow{r_{1}}, L\right) U_{0}^{*}\left(\overrightarrow{r_{2}}, L\right)\left\langle\exp \left[\psi\left(\overrightarrow{r_{1}}, L\right)+\psi^{*}\left(\overrightarrow{r_{2}}, L\right)\right]\right\rangle, \\
\sigma_{r}^{2}(r, L) & =2 \pi^{2} k^{2} L \int_{0}^{1} \int_{0}^{\infty} \kappa \Phi_{n}(\kappa) \exp \left(-\frac{\Lambda L \kappa^{2} \xi^{2}}{k}\right)\left[I_{0}(2 \Lambda r \xi \kappa)-1\right] d \kappa d \xi, \\
\sigma_{r}^{2}(0, L) & =2 \pi^{2} k^{2} L \int_{0}^{1} \int_{0}^{\infty} \kappa \Phi_{n}(\kappa) \exp \left(-\frac{\Lambda L \kappa^{2} \xi^{2}}{k}\right)\left\{1-\cos \left[\frac{L \kappa^{2}}{k} \xi(1-\bar{\Theta} \xi)\right]\right\} d \kappa d \xi,
\end{aligned}
$$

and $I_{0}(\cdot)$ is the modified Bessel function of zero order, $T$ is a term denoting the fluctuations of on-axis mean irradiance at the receiver plane caused by atmospheric turbulence (see Andrews \& Philips, 2005, Chap. 6.3), and the most-right exponential of Eq. (35) is the complex degree of coherence (DOC).

From the MCF and the DOC physical effects on the optical traveling wave can be derived, namely, the mean irradiance, turbulence-induced beam spreading, angle-of-arrival fluctuations and beam wander.

Actually, the most noticeable effect caused by atmospheric turbulence is the optical scintillation, and it is quantified by the scintillation index (SI)

$$
\sigma_{I}^{2}(\vec{r}, L)=\frac{\left\langle I^{2}(\vec{r}, L)\right\rangle}{\langle I(\vec{r}, L)\rangle^{2}}-1,
$$

where $I(\vec{r}, L)$ denotes the irradiance of the optical field in the receiver plane.

The mathematical derivation of the SI relies upon the fourth statistical moment of the optical field $U(\vec{r})$, given by

$$
\Gamma_{4}\left(\overrightarrow{r_{1}}, \overrightarrow{r_{2}}, \overrightarrow{r_{3}}, \overrightarrow{r_{4}}, L\right)=\left\langle U\left(\overrightarrow{r_{1}}, L\right) U^{*}\left(\overrightarrow{r_{2}}, L\right) U\left(\overrightarrow{r_{3}}, L\right) U^{*}\left(\overrightarrow{r_{4}}, L\right)\right\rangle
$$

By setting $\overrightarrow{r_{1}}=\overrightarrow{r_{2}}=\overrightarrow{r_{3}}=\overrightarrow{r_{4}}=\vec{r}$ and evaluating Eq. (32) and Eq. (38) for the same point, yields

$$
\begin{aligned}
\left\langle I^{2}(\vec{r}, L)\right\rangle & =\Gamma_{4}(\vec{r}, \vec{r}, \vec{r}, \vec{r}, L), \\
\langle I(\vec{r}, L)\rangle & =\Gamma_{2}(\vec{r}, \vec{r}, L),
\end{aligned}
$$

thus, obtaining a theoretical expression for the scintillation index.

A fundamental parameter in the study of optical wave propagation through random media is the Rytov variance $\sigma_{R}^{2}$, which is in fact the scintillation index for a plane wave in the weak turbulence regime. The Rytov variance can be derived from Eq. (36), and by setting $\Lambda=0$ and $\Theta=1$ in the limiting case of a plane wave, yields to

$$
\sigma_{R}^{2}=1.23 C_{n}^{2} k^{7 / 6} L^{11 / 6} .
$$

A more detailed explanation on the derivation of the solution of Eq. (10), and the statistical moments of the optical field can be found in (Andrews \& Philips, 2005). 


\subsection{Extended Rytov theory}

The Rytov approximation is valid only in weak irradiance fluctuations regime, and an extension of the theory is needed to address stronger turbulence effects on optical traveling waves. As a wave propagates through the turbulent atmosphere its degree of transverse spatial coherence decreases, this coherence lost is quantified by the spatial coherence radius

$$
\rho_{0}= \begin{cases}\left(\frac{3}{1+\Theta+\Theta^{2}+\Lambda^{2}}\right)^{1 / 2}\left(1.87 C_{n}^{2} k^{2} L l_{0}^{-1 / 3}\right)^{-1 / 2}, & \rho_{0} \ll l_{0} \\ \left(\frac{8}{3\left(a+0.62 \Lambda^{11 / 6}\right)}\right)^{3 / 5}\left(1.46 C_{n}^{2} k^{2} L\right)^{-3 / 5}, & l_{0} \ll \rho_{0} \ll L_{0},\end{cases}
$$

where $a$ is a constant (see Andrews \& Philips, 2005, p. 192). It should be noted that $\Theta$ and $\Lambda$ are dimentionless parameters associated with the Gaussian beam. The expression for $\rho_{0}$ in the limiting cases of a plane wave $(\Lambda=0, \Theta=1)$, and a spherical wave $(\Lambda=0, \Theta=0)$ can be deduced form Eq. (42).

Another parameter to measure the spatial coherence is the atmospheric coherence width $r_{0}=$ $2.1 \rho_{0}$, widely known as the Fried parameter. For the limiting case of a plane wave the Fried parameter is given by

$$
r_{0}=\left(0.42 C_{n}^{2} k^{2} L\right)^{-3 / 5} .
$$

Under the extended Rytov theory the refractive-index $n_{1}(\vec{r})$ in Eq. (1) can be seen as the result of the influence of two terms, i.e., the large-scale inhomogeneities $n_{X}(\vec{r})$ and the small-scale inhomogeneities $n_{Y}(\vec{r})$. Thus, as the refractive-index directly influences the turbulence power spectrum, an effective power spectral density for refractive-index fluctuations can be expressed by

$$
\Phi_{n e}(\kappa)=\Phi_{n}(\kappa) G\left(\kappa, l_{0}, L_{0}\right)=\Phi_{n}(\kappa)\left[G_{X}\left(\kappa, l_{0}, L_{0}\right)+G_{Y}\left(\kappa, l_{0}\right)\right],
$$

where $G_{X}$ and $G_{Y}$ are amplitude spatial filters modeling the large-scale and small-scale perturbations, respectively.

The effective atmospheric spectrum can be used instead of the classic spectrum to solve the statistical moments of a traveling optical field, thus, allowing to treat the effects of inner-scale size and outer-scale size of turbulence separately throughout the theory.

\subsection{Physical effects}

\subsubsection{Angle-of-arrival fluctuations}

Referring to Fig. 1, the rays (solid arrows) leaving the laser source are deflected as they travel through the turbulent atmosphere, some arriving off-axis instead of what is expected without turbulence, represented with the horizontally straight dashed arrow. As the rays may also be interpreted as the wave vector for the traveling wavefront, the variations in the angle respect the optical axis at the receiver represent the concept of angle-of-arrival fluctuations. The expression for the angle-of-arrival fluctuations, that directly depends on the turbulence strength and the optical path length, is given by

$$
\left\langle\beta_{a}^{2}\right\rangle=2.91 C_{n}^{2} L\left(2 W_{G}\right)^{-1 / 3},
$$

where $W_{G}$ is soft aperture radius, and it is related to the receiving aperture $D$ by $D^{2}=8 W_{G}^{2}$. 
The main technique to counterbalance the degrading effects of receiving the optical wave off-axis, is by the combination of fast steering mirrors and adaptive optics algorithms (Levine et al., 1998; Tyson, 2002; Weyrauch \& Vorontsov, 2004).

\subsubsection{Beam wander}

The beam wander effect is related with the displacement of the instantaneous center of the beam-defined as the point of maximum irradiance-of a traveling wave over the receiver plane. It is well known that this phenomenon is caused by the large-scale inhomogeneities due to their refractive effects. A Gaussian beam wave after propagating through the turbulent atmosphere is corrupted in such a way that the instantaneous field, at the receiver plane, greatly differs of what if expected for a Gaussian beam, with the added characteristic that the beam center can exhibit great deviation from the optical axis of the optical link.

Instead, the short-term and long-term fields have a field shape that resembles that of a Gaussian beam. Nevertheless, the optical field in the short-term exposition is skewed from a Gaussian beam profile, while the long-term profile describes a more accurately Gaussian profile and the deviation of the beam center from the optical axis is relatively small, and can be neglected. A computer simulation of a Gaussian beam, shown in Fig. 4, was conducted following the method described in Dios et al. (2008), where the field profile for different exposure times is presented. For this simulation it was assumed a propagation distance of $L=2000 \mathrm{~m}, C_{n}^{2}=0.6 \cdot 10^{-14} \mathrm{~m}^{-2 / 3}, \lambda=1064 \mathrm{~nm}, W_{0}=2 \mathrm{~cm}$, and the exposition time of the long-term profile in Fig. 4(c) is 34 times of that used for the short-term profile in Fig. 4(b).

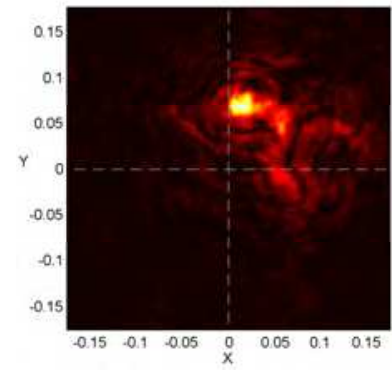

(a) Instantaneous beam profile.

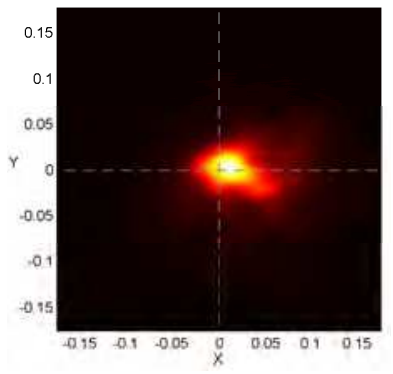

(b) Short-term beam profile.

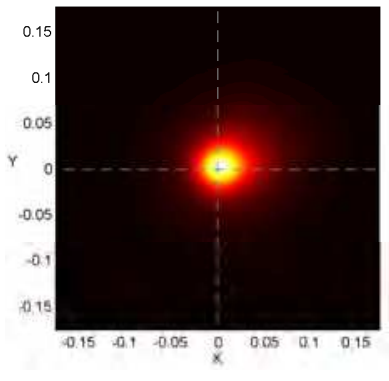

(c) Long-term beam profile.

Fig. 4. Profile of a Gaussian beam with different exposure times, after propagating .

Fante (1980) in his work relate the random displacements of the incoming wavefront center or "hot spot" with the long-term $W_{\mathrm{LT}}$ and short-term $W_{\mathrm{ST}}$ spot sizes, assuming that the "hot spot" coincides with beam centroid, by the expression

$$
\left\langle r_{c}^{2}\right\rangle=W_{\mathrm{LT}}^{2}-W_{\mathrm{ST}}^{2}
$$

where $W_{L T}$ is the long-term beam radius, and following a conventional Rytov method, its form is (Recolons et al., 2007)

$$
W_{\mathrm{LT}}^{2}=W^{2}\left[1+1.33 \sigma_{R}^{2} \Lambda^{5 / 6}\right], \quad \Lambda=\frac{2 L}{k W^{2}},
$$


where $W$ is the pure diffraction beam radius at the receiver plane. Furthermore, the short-term beam radius is given by

$$
W_{\mathrm{ST}}^{2}=W^{2}\left\{1+1.33 \sigma_{R}^{2} \Lambda^{5 / 6}\left[1-0.66\left(\frac{\Lambda_{0}^{2}}{1+\Lambda_{0}^{2}}\right)^{1 / 6}\right]\right\} .
$$

It is clear how in Eq. (47) and Eq. (48) the extra spreading effect due to the atmospheric turbulence is included through the Rytov variance $\sigma_{R}^{2}$.

For the sake of simplicity, the geometrical optics approximation used by Churnside \& Lataitis (1990) yields to a closed form expression for the beam wander

$$
\left\langle r_{c}^{2}\right\rangle=0.97 C_{n}^{2} L^{3} W_{0}^{-1 / 3},
$$

while taking into account that this expression is valid for an infinite outer scale and a collimated beam, as is mostly assumed.

\subsubsection{Scintillation}

A laser beam propagating through the atmosphere will be altered by refractive-index inhomogeneities. At the receiver plane, a random pattern is produced both in time and space (Churnside, 1991). The irradiance fluctuations over the receiver plane resemble the speckle phenomenon observed when a laser beam impinges over a rugged surface. The parameter that express these irradiance fluctuations is the scintillation index

$$
\sigma_{I}^{2}=\frac{\left\langle I^{2}\right\rangle-\langle I\rangle^{2}}{\langle I\rangle^{2}}=\frac{\left\langle I^{2}\right\rangle}{\langle I\rangle^{2}}-1
$$

where $I \equiv I(0, L)$ denotes irradiance of the optical wave observed by a point detector after propagating a distance $L$.

Classical studies on optical wave propagation have been classified in two major categories, either the weak or strong fluctuations theory. It is customary to discriminate both cases for a given propagation problem by determining the value of the Rytov variance. The weak fluctuations regime occurs when $\sigma_{R}^{2}<1$, the strong fluctuations regime is associates with $\sigma_{R}^{2}>$ 1 , while there is the saturation regime when $\sigma_{R}^{2} \rightarrow \infty$. Different expression are derived for the SI depending on whether the calculation have to be done in the weak or the strong fluctuations regime, although, when $\sigma_{R}^{2} \sim 1$ both expression will give similar results. Andrews et al. (2001) have developed a set of expressions for the SI of Gaussian-beam waves and claimed to be valid in the weak-to-strong fluctuation regime. This work is based on the extended Rytov theory in combination with the solution of the Helmholtz equation given by Eq. (29). The idea behind this approach is to separate the influence of the turbulence in two parts, namely, that caused by the small-scale eddies - that are assumed to be diffractive inhomogeneities_-on one hand, and, on the other hand, the effects caused by the large-scale eddies-regarded as refractive inhomogeneities. Mathematically the irradiance is then written as

$$
\hat{I}=\frac{I}{\langle I\rangle}=X Y,
$$


where $X$ and $Y$ are unit mean statistically independent processes arising from the large-scale and small-scale size of turbulence, respectively. Alternatively, the irradiance can be written as $I=A \exp (2 \chi)$, where $\chi$ is the log-amplitude of the optical wave. Moreover, when $\chi$ is normally distributed, the variance of the log-amplitude is related to scintillation index according to

$$
\sigma_{I}^{2}=\exp \left(4 \sigma_{\chi}^{2}\right)-1=\exp \left(\sigma_{\ln I}^{2}\right)-1
$$

where $\sigma_{\ln I}^{2}$ is the variance of the log-irradiance, that in turn depends on the large-scale $\sigma_{\ln X}^{2}$ and small-scale $\sigma_{\ln Y}^{2}$ variances, as follows

$$
\sigma_{\ln I}^{2}=4 \sigma_{\chi}^{2}=\sigma_{\ln X}^{2}+\sigma_{\ln Y}^{2}
$$

On the other hand, the on-axis scintillation index for a point receiver takes the integral form

$$
\sigma_{I}^{2}=8 \pi^{2} k^{2} L \int_{0}^{1} \int_{0}^{\infty} \kappa \Phi_{n}(\kappa) \exp \left(-\frac{\Lambda L \kappa^{2} \xi^{2}}{k}\right)\left\{1-\cos \left[\frac{L \kappa^{2}}{k} \xi(1-\bar{\Theta} \xi)\right]\right\} d \kappa d \xi
$$

where $\bar{\Theta}=1-\Theta$, and $\Phi_{n}(\kappa)$ can be replaced by the effective spectrum in Eq. (44) in order to account for the effects produced by the large-scale $G_{X}$ and small-scale perturbations $G_{Y}$, defined by

$$
\begin{aligned}
& G_{X}(\kappa)=\exp \left(\frac{\eta}{\eta_{X}}\right), \eta=\frac{L \kappa^{2}}{k}, \\
& G_{Y}(\kappa)=\frac{\kappa^{11 / 3}}{\left(\kappa^{2}+\kappa_{Y}^{2}\right)^{11 / 6}} \exp \left[\frac{\Lambda L \kappa^{2}(1-z / L)^{2}}{k}\right], \kappa_{Y} \gg 1,
\end{aligned}
$$

where $\eta_{X}$ and $\kappa_{Y}$ are variables including the refractive-index structure parameter and some Gaussian beam parameters (see Andrews \& Philips, 2005, Sec. 9.6.2). It should be noted that Eq. (55) does not account for inner-scale and outer-scale of turbulence effects.

It is noteworthy that under weak turbulence regime Eq. (52) and Eq. (53) yields to $\sigma_{I}^{2} \cong$ $\sigma_{\ln I}^{2}=4 \sigma_{\chi}^{2}=\sigma_{\ln X}^{2}+\sigma_{\ln \gamma}^{2}$, and following the strategy described above, combining Eq. (44) and Eq. (55) with Eq. (54), the optical scintillation index on-axis of a Gaussian beam for a point receiver is (Andrews et al., 2001)

$$
\sigma_{I}^{2}=\exp \left(\sigma_{\ln X}^{2}+\sigma_{\ln Y}^{2}\right)-1=\exp \left\{\frac{0.49 \sigma_{B}^{2}}{\left[1+0.56(1+\Theta) \sigma_{B}^{12 / 5}\right]^{7 / 6}}+\frac{0.51 \sigma_{B}^{2}}{\left(1+0.69 \sigma_{B}^{12 / 5}\right)^{5 / 6}}\right\}-1 .
$$

where $\sigma_{B}^{2}$ is the Rytov variance for a Gaussian beam wave, and it is given by

$$
\sigma_{B}^{2}=3.86 \sigma_{R}^{2}\left\{0.40\left[(1+2 \Theta)^{2}+4 \Lambda^{2}\right]^{5 / 12} \cos \left[\frac{5}{6} \tan ^{-1}\left(\frac{1+2 \Theta}{2 \Lambda}\right)\right]-\frac{11}{16} \Lambda^{5 / 6}\right\},
$$

where $\Theta$ and $\Lambda$ are defined by Eq. (20) and Eq. (21), respectively. 
On the other hand, the expression of the scintillation index for a receiver over a finite aperture $D$ is given by

$$
\begin{aligned}
\sigma_{I}^{2}(D)= & 8 \pi^{2} k^{2} L \int_{0}^{1} \int_{0}^{\infty} \kappa \Phi_{n}(\kappa) \exp \left(-\frac{L \kappa^{2}}{k\left(\Lambda+\Omega_{G}\right)}\left[(1-\bar{\Theta} \xi)^{2}+\Lambda \Omega_{G} \xi^{2}\right]\right) \\
& \times\left(1-\cos \left[\frac{L \kappa^{2}}{k}\left(\frac{\Omega_{G}-\Lambda}{\Omega_{G}+\Lambda}\right) \xi(1-\bar{\Theta} \xi)\right]\right) d \kappa d \xi, \quad \Omega_{G} \geq \Lambda,
\end{aligned}
$$

where $\Omega_{G}=2 L / k W_{G}$ is a non-dimensional parameter defining the beam radius at the colleting aperture element. A tractable expression for Eq. (58) have been derived, based on the large-scale and small-scale variances (Andrews \& Philips, 2005). Following Eq. (53)

$$
\sigma_{I}^{2}(D)=\exp \left(\sigma_{\ln X}^{2}(D)+\sigma_{\ln Y}^{2}(D)\right)-1 .
$$

where large-scale and small-scale log-irradiance variances are given by

$$
\begin{gathered}
\sigma_{\ln X}^{2}(D)=\frac{0.49\left(\frac{\Omega_{G}-\Lambda}{\Omega_{G}+\Lambda}\right)^{2} \sigma_{B}^{2}}{\left[1+\frac{0.4(2+\Theta)\left(\sigma_{B} / \sigma_{R}\right)^{12 / 7}}{\left(\Omega_{G}+\Lambda\right)\left(\frac{1}{3}-\frac{1}{2} \bar{\Theta}+\frac{1}{5} \bar{\Theta}^{2}\right)^{6 / 7}}+0.56(1+\Theta) \sigma_{B}^{12 / 5}\right]^{7 / 6}}, \\
\sigma_{\ln Y}^{2}(D)=\frac{0.51 \sigma_{B}^{2}\left(\Omega_{G}+\Lambda\right)\left(1+0.69 \sigma_{B}^{12 / 5}\right)^{-5 / 6}}{\Omega_{G}+\Lambda+1.20\left(\sigma_{R} / \sigma_{B}\right)^{12 / 5}+0.83 \sigma_{R}^{12 / 5}} .
\end{gathered}
$$

It should be noticed that these expressions do not account for the inner and outer scale of turbulence effects. In order to include them, additional considerations must be made when developing Eq. (53) as shown in Andrews et al. (2001).

In Fig. 5 a plot of the scintillation index is shown, where different receiving aperture diameters have been used to calculate Eq. (59), and a collimated Gaussian beam have been assumed with wavelength $\lambda=780 \mathrm{~nm}$ and beam size at the transmitter $W_{0}=1.13 \mathrm{~cm}$. Additionally, the effects of the inner scale and the outer scale of turbulence were neglected, and the transmitter aperture diameter was set to $3 \mathrm{~cm}$.

From the analysis of the Fig. 5 it can be conclude that a wireless optical communication link can be classified in one of three well differentiated zones. In the first one, regarded as the weak turbulence regime, the scintillation index increases monotonically as either the optical turbulence, denoted by the refractive-index structure parameter, or the link distance increases. Next, a peak in the scintillation index appear representing the point of maximum atmospheric turbulence. This zone is known as the strong turbulence regime. Finally, a third zone called the saturation regime settles the value of the scintillation index to a plateau. The physical reason for the constant level of the SI, irrespective of the increase of the $C_{n}^{2}$ value or the link range, is because after a certain point the atmospheric turbulence completely breaks the spatial coherence of the traveling wavefront and the arriving optical power behaves as a diffuse source. It becomes evident that the localization of the three turbulence regimes explained before is affected by the size of the receiving aperture size. 


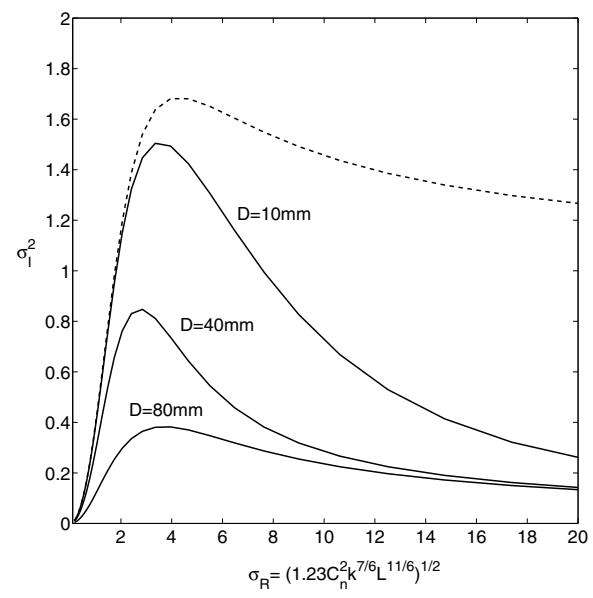

Fig. 5. Scintillation index for different receiver aperture diameters. As reference the SI for a point receiver is shown (dashed line).

\subsubsection{Aperture averaging}

To counterbalance the scintillation effects on the optical link performance, it is desirable to have a large area at the detection plane in order to integrate as much light as possible. The received wavefront can be regarded as a self-interference pattern, produced by atmospheric inhomogeneities of different spatial scale sizes, that is averaged over the entire receiving area, thus, the intensity fluctuations are mitigated. From the ray optics point of view more rays, which all travel through distinct optical paths, can be collected by means of a lens to be integrated on the photodetector and the measured scintillation index will be lesser compared to that of a point receiver. This phenomenon, called aperture averaging, have been extensively addressed (Andrews, 1992; Churnside, 1991; Fried, 1967). Churnside (1991) developed simple closed expressions to easily evaluate aperture averaging under weak fluctuation regime, that were later corrected by Andrews (1992). More recently, expression for aperture averaging of Gaussian beams have developed for the moderate-to-strong turbulences regime (Andrews et al., 2001; Andrews \& Philips, 2005).

The mathematical expression for the aperture averaging factor $A$ is defined by

$$
A(D)=\frac{\sigma_{I}^{2}(D)}{\sigma_{I}^{2}(0)},
$$

where $\sigma_{I}^{2}(D)$ is the SI of a receiving aperture with diameter $D$, and $\sigma_{I}^{2}(0)$ is the SI for a point receiver defined in Eq. (56). As far as experimental data is concerned an appropriate way to evaluate the aperture averaging factor is by using an effective point receiver, defined as an aperture much smaller than $\sqrt{\lambda L}$ and the inner scale size $l_{0}$ (Ochs \& Hill, 1985). It is noteworthy that the lowest possible value of $A$ is desirable, in order to overcome signal fluctuations due to atmospheric turbulence.

In Fig. 6 the aperture averaging factor is shown for Gaussian beam with the same characteristics of that used to plot Fig. 5. Additionally, a link distance of $2 \mathrm{~km}$ was set. It is clear that for higher turbulence strength the aperture averaging effect becomes less noticeable. 


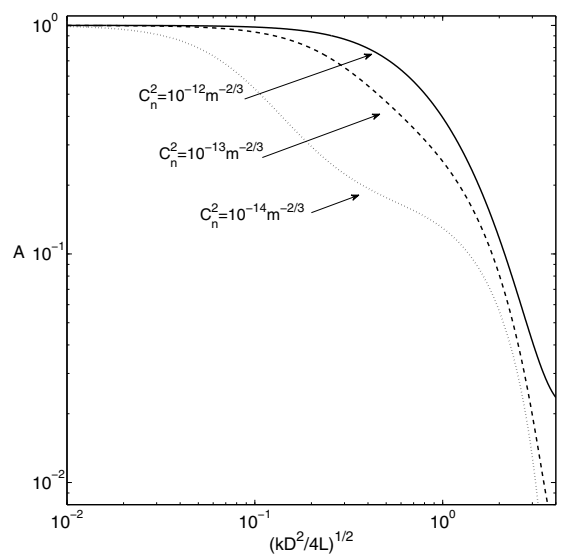

Fig. 6. Aperture averaging factor for different atmospheric turbulence conditions as a function of the receiving aperture radius $D / 2$ normalized to the Fresnel zone $\sqrt{L / k}$.

Moreover, it is evident that the averaging capability of the receiver system increases as the the receiving aperture diameter increases.

\section{Propagation simulation}

Since its introduction by Fleck et al. (1976) the beam split-step method has been widely used to simulate the propagation of electromagnetic waves, where the effects produced by the turbulent atmosphere are simulated by a series of linearly spaced random phase screens. In Fig. 7 are depicted the main aspects involved in the beam split-step method, also known as the beam propagation method (BPM). First, an initial traveling optical field is set and the path length $L$ is split into a series of $N$ steps, thus, dividing the optical path into $N$ different slabs of turbulent atmosphere of width $\Delta z=L / N$. Each of this slabs is represented by a two-dimensional (2D) random phase screen placed in the middle of such slab. Consequently, the first and last propagation step have length $\Delta z / 2$ while all other steps are $\Delta z$ in length. The propagation of the optical field between every step takes place in the transformed domain, where the field is decomposed into a linear combination of plane waves. After each step the optical wavefront is inverse transformed, to the spatial domain, where a random phase screen is then used to simulate the atmospheric effects. This process is repeated until the propagation path length is completed.

At the receiver end the detector is a single pixel in the case of a point receiver. When considering a finite size receiving aperture, the optical power in the two-dimensional grid of the traveling wavefront is integrated over the aperture area.

The most widespread technique used to generate the random phase screens is based on the spectral method, in which phase screens are generated in the spectral domain with the selected turbulence power spectrum (Frehlich, 2000; Martin \& Flattè, 1998; Recolons \& Dios, 2005). The fractal method is an alternative approach to reproduce the phase screens directly in the spatial domain by successive interpolations from a set of random numbers that obey the desired 


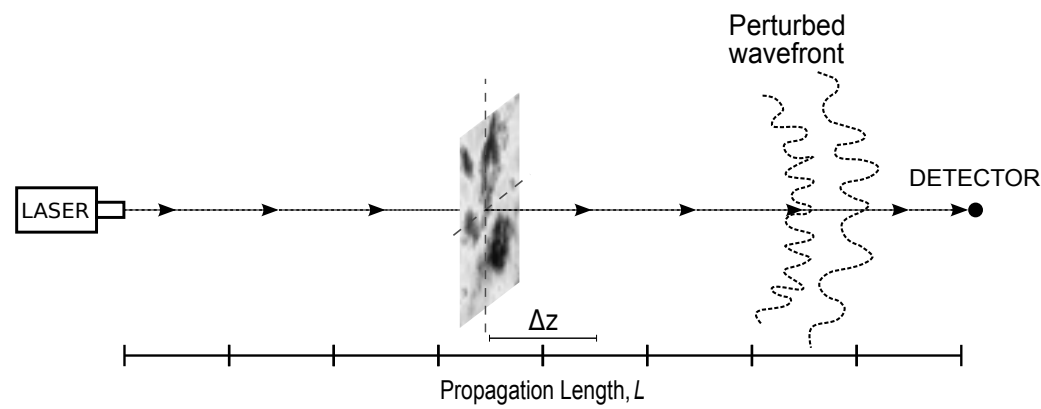

Fig. 7. General scheme of beam propagation method (BPM) applied to the propagation of light through the turbulent atmosphere.

structure function associated with the turbulence power spectrum being used (Lane et al., 1992).

\subsection{Spectral method}

In this method the screens are numerically generated by the use of fast Fourier-transform and assumed to follow a particular turbulence power spectrum, which most employed is the Kolmogorov spectrum.

The phase screen is generated in the spectral domain by means of filtering Gaussian white noise with the selected turbulence power spectrum, and an inverse transformation yields to the desired random phase screen in the spatial domain, which is given by (Frehlich, 2000)

$$
\theta_{s}(j \Delta x, l \Delta y)=\sum_{n=0}^{N_{x}} \sum_{m=0}^{N_{y}}[a(n, m)+i b(n, m)] \times \exp [2 \pi i(j n / N x+l m / N y)],
$$

where $i=\sqrt{-1} ; \Delta x$ and $\Delta y$ are the grid spacing; $N_{x}$ and $N_{y}$ are the number of points in the respective dimension of the screen; and $a(n, m)$ and $b(n, m)$ are random number following Gaussian white noise statistics with

$$
\left\langle a^{2}(n, m)\right\rangle=\left\langle b^{2}(n, m)\right\rangle=\frac{8 \pi^{3} k^{2} \Delta z}{N_{x} \Delta x N_{y} \Delta y} \Phi_{n}(\vec{\kappa}, z),
$$

where $k$ is the wave number, $\Phi_{n}(\vec{\kappa}, z)$ is the two-dimensional power spectrum for refractive-index fluctuations as a function of the propagation distance $z$, and $\vec{\kappa}$ is the spatial wave number vector in the plane transversal to propagation direction.

However, a major difficulty with this technique is to reproduce atmospheric large-scale effects owing to the fact that they are related with lowest spatial frequencies of the turbulence spectrum, and, it is precisely around zero where the Kolmogorov spectrum have a singularity. This issue was addressed first by Lane et al. (1992) with the addition of subharmonic components to the random phase screen, as a result of which more resolution in the spatial frequencies around zero is obtained. Later, an improved version of this method was introduced by Recolons \& Dios (2005). 


\subsection{Fractal method}

Phase screens generated following a Kolmogorov power spectrum have an important property, namely, that they present a fractal behavior as they look similar regardless of the scale they are viewed. The first to propose the use of fractal interpolation for generating phase screens was Lane et al. (1992), and later an improved version of this method was introduced (Harding et al., 1999).

With this method, first, an exact low-resolution phase screen is generated by evaluating its covariance matrix that is obtained directly from the structure function. Which for a pure Kolmogorov spectrum, as it is normally assumed, is given by

$$
D\left(\overrightarrow{r_{1}}, \overrightarrow{r_{2}}\right)=\left\langle\left[\theta_{s}\left(\overrightarrow{r_{1}}\right)-\theta_{s}\left(\overrightarrow{r_{2}}\right)\right]^{2}\right\rangle=6.88\left(\frac{\left|\overrightarrow{r_{1}}-\overrightarrow{r_{2}}\right|}{r_{0}}\right)^{5 / 3},
$$

where $\theta_{S}\left(\overrightarrow{r_{1}}\right)$ and $\theta_{S}\left(\overrightarrow{r_{2}}\right)$ are the phase evaluated at positions $\overrightarrow{r_{1}}$ and $\overrightarrow{r_{2}}$, respectively, and $r_{0}$ is the Fried parameter. The covariance matrix can be obtained from the structure function with the relationship

$$
\begin{aligned}
C_{\theta_{s}}\left(\overrightarrow{r_{1}}, \overrightarrow{r_{2}}\right)= & -\frac{1}{2} D\left(\overrightarrow{r_{1}}, \overrightarrow{r_{2}}\right)+\frac{1}{2} \iint D\left(\vec{r}_{1}^{\prime}, \overrightarrow{r_{2}}\right) T\left({\overrightarrow{r_{1}}}^{\prime}\right) d x_{1}^{\prime} d y_{1}^{\prime} \\
& +\frac{1}{2} \iint D\left(\overrightarrow{r_{1}},{\overrightarrow{r_{2}}}^{\prime}\right) T\left({\overrightarrow{r_{2}}}^{\prime}\right) d x_{2}^{\prime} d y_{2}^{\prime} \\
& -\frac{1}{2} \iiint \int D\left({\overrightarrow{r_{1}}}^{\prime},{\overrightarrow{r_{2}}}^{\prime}\right) T\left(\vec{r}_{1}^{\prime}\right) T\left({\overrightarrow{r_{2}}}^{\prime}\right) d x_{1}^{\prime} d y_{1}^{\prime} d x_{2}^{\prime} d y_{2}^{\prime},
\end{aligned}
$$

where $T(\vec{r})$ is a windowing function that has a constant value inside the dominion of the phase screens and zero value outside. Next, once the covariance matrix is obtained, an square matrix matching the size of the phase screen is generated from a set of Gaussian random numbers with variance given by the eigenvalues of $C_{\theta_{s}}\left(\overrightarrow{r_{1}}, \overrightarrow{r_{2}}\right)$ (Harding et al., 1999). Nevertheless, is important to note that if the initial squared phase screen have a size $N \times N$, then the covariance matrix has $N^{2} \times N^{2}$ dimension, making the method applicable to small values of $N$. Thereby, the use of interpolation techniques rises as mandatory to obtain phase screen with higher resolution. Probably, the most widespread window sizes in the literature are $N=512$ and $N=1024$.

When the low-resolution phase screen have been completely generated, successive randomized interpolation steps are executed to produce the desired grid size. This interpolation method achieve a high resolution degree while demanding a relatively small computational effort, although, having the drawback of poorer statistical performance.

\section{Wireless optical communication systems}

Previous sections have been focused on the explanation of the physical phenomena that affects an optical traveling wave in a free-space optical link, as shown in Fig. 1. From a communication system approach, there are other factors that become critical when evaluating the performance of a wireless optical communications link. A simplified scheme is shown in Fig. 8, where the main factors involved in are presented.

Wireless optical communications rely on a traveling wave generated by a laser source, at certain average power level transmitted $P_{T}$. Aside from the effects suffered by the optical 


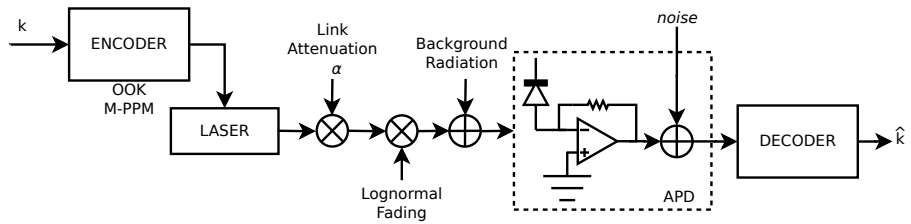

Fig. 8. Block diagram for a wireless optical communication link.

traveling wavefront through the turbulent atmospheric channel, addressed in Section 3, the average optical power at the receiver plane $P_{R}$ is influenced by various parameter. The expression for the average optical power detected at a distance $R$ in a WOC link, is given by

$$
P_{R}(R)=P_{T} \frac{D_{R}^{2}}{D_{T}^{2}+(R \theta)^{2}} \exp \left(-\frac{\theta_{m p}^{2}}{(\theta / 2)^{2}}\right) T_{a}(R) T_{R},
$$

where $\theta$ is the laser beam full-angle divergence, $T_{a}(R)$ is the transmittance of the atmosphere along the optical path, $T_{R}$ is the transmittance of the receiver optics, $\theta_{m p}$ denotes pointing errors between the emitter and receiver, and, $D_{T}$ and $D_{R}$ are the transmitting and receiving apertures diameters, respectively. It should be noted that pointing errors not only are due to misalignments in the installation process, but also to vibrations on the transmitter and receiver platforms. For horizontal links the vibration come from transceiver stage oscillations and buildings oscillations caused by wind, while for vertical links-i.e. ground to satellite link - satellite wobbling oscillation are the main source of pointing errors.

\subsection{Atmospheric attenuation}

A laser beam traveling through the turbulent atmosphere is affected by extinction due to aerosols and molecules suspended in the air. The transmittance of the atmosphere can be expressed by Beer's law as

$$
T_{a}(R)=\frac{P(R)}{P(0)}=e^{-\alpha_{a} R},
$$

where $P(0)$ is transmitted laser power at the source, and $P(R)$ is the laser power at a distance $R$. The total extinction coefficient per unit length $\alpha_{a}$ comprises four different phenomena, namely, molecular and aerosol scattering , and, molecular and aerosol absorption:

$$
\alpha_{a}=\alpha_{a b s}^{m o l}+\alpha_{a b s}^{a e r}+\beta_{s c a}^{m o l}+\beta_{s c a}^{a e r} .
$$

The molecular and aerosol behavior for the scattering and absorption process is wavelength dependent, thus, creating atmospheric windows where the transmission of optical wireless signal is more favored. The spectral transmittance of the atmosphere is presented in Fig. 9, for a horizontal path of nearly $2 \mathrm{~km}$ at sea level (Hudson, 1969).

Within the atmospheric transmittance windows the molecular and aerosol absorption can be neglected. Molecular scattering is very small in the near-infrared, due to dependence on $\lambda^{-4}$, and can also be neglected. Therefore, aerosol scattering becomes the dominating factor reducing the total extinction coefficient to (Kim et al., 1998)

$$
\alpha_{a}=\beta_{\text {sca }}^{a e r}=\frac{3.91}{V}\left(\frac{\lambda}{550}\right)^{-q},
$$




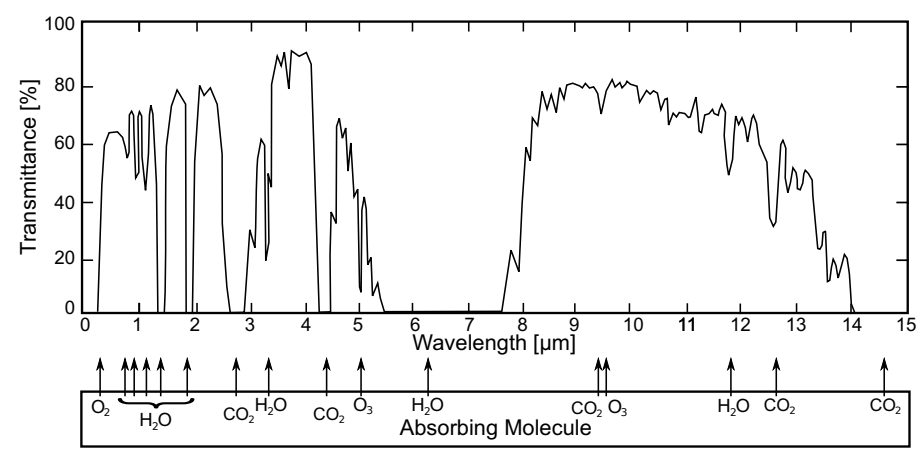

Fig. 9. Earth's atmospheric transmittance [Adapted from Hudson (1969)].

where $V$ is the visibility in kilometers, $\lambda$ is the wavelength in nanometers, and $q$ is the size distribution of the scattering particles. Typical values for $q$ are given in Table 3.

The attenuation factors that supposed the larger penalties are the atmospheric attenuation and the geometrical spreading losses, both represented in Fig. 10. It becomes evident from the inspection of their respective behaviors, that the atmospheric attenuation imposes larger attenuation factors for poor visibility conditions than the geometrical losses due to the beam divergence of the laser source. Meteorological phenomena as snow and haze are the worst obstacle to set horizontal optical links, and, of course, the clouds in vertical ground-to-satellite links, which imposed the need of privileged locations for deploying optical ground stations.

\begin{tabular}{lc}
\hline \hline Visibility & $q$ \\
\hline$V>50 \mathrm{~km}$ & 1.6 \\
$6 \mathrm{~km}<V<50 \mathrm{~km}$ & 1.3 \\
$V<6 \mathrm{~km}$ & $0.585 V^{1 / 3}$ \\
\hline \hline
\end{tabular}

Table 3. Value of the size distribution of the scattering particles $q$, for different visibility conditions.

For the calculations in Fig. 10(a) a light source with wavelength $\lambda=780 \mathrm{~nm}$ was assumed, and for Fig. 10(b) the aperture diameter in transmission and reception was set to $4 \mathrm{~cm}$ and $15 \mathrm{~cm}$, respectively. The negative values of the attenuation in Fig. 10(b) imply that the geometrical spreading, of the transmitted beam, have not yet exceeded in size the receiving aperture.

\subsection{Background radiance}

In a wireless optical communication link the receiver photodetector is always subject to an impinging optical power, even when no laser pulse have been transmitted. This is because the sun radiation is scattered by the atmosphere, the Earth's surface, buildings, clouds, and water masses, forming a background optical power. The amount of background radiance detected in the receiver depends on the area and the field of view of the collecting telescope, the optical bandwidth of the photodetector, and weather conditions. The most straightforward method to decrease background radiation is by adding an interference filter with the smallest possible optical bandwidth, and the center wavelength matching that of the laser source. Typical values of optical bandwidth these filters are in orders of a few nanometers. 


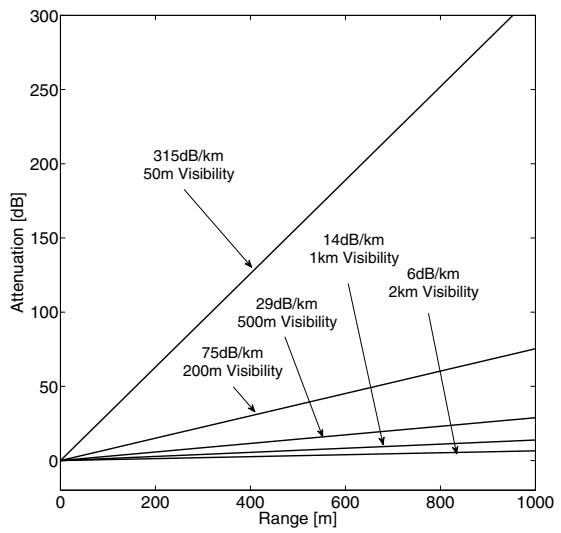

(a) Atmospheric attenuation in decibels for light source with $\lambda=780 \mathrm{~nm}$ and various visibility conditions.

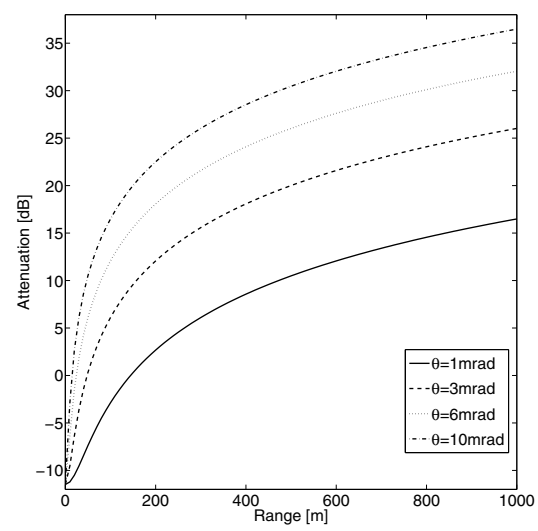

(b) Geometrical spreading loss in decibels for various beam full-angle divergences of light source.

Fig. 10. Attenuation factor dependence on link distance.

The total background radiation can be characterized by the spectral radiance of the sky, which is different for day or night operation. The curves for daytime conditions will be very similar to those of nighttime, with the addition of scattered sun radiation below $3 \mu \mathrm{m}$ (Hudson, 1969). The typical behavior of the spectral radiance of the sky is shown in Fig. 11 for daytime condition and an horizontal path at noon.

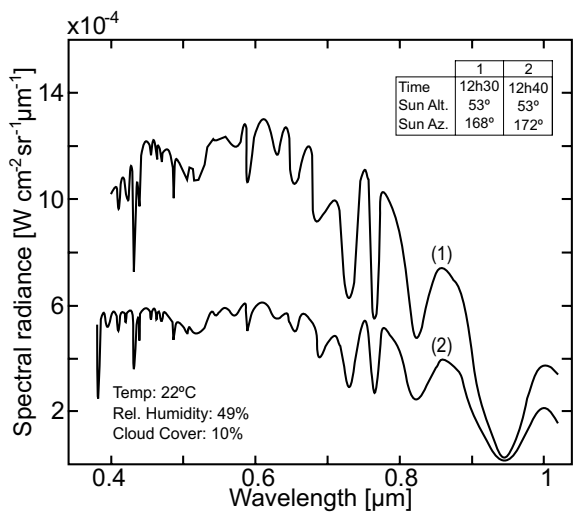

Fig. 11. Spectral radiance of the sky for a clear daytime [Adapted from Knestrick \& Curcio (1967)].

Once the spectral radiance of the sky is known the total optical power at the receiver, due to background, can be calculated by

$$
P_{B}=N_{B} T_{R}\left(\pi \frac{D}{2} \frac{\mathrm{FOV}}{2}\right)^{2} B_{\text {opt }},
$$


where $N_{B}$ is the background spectral radiance, FOV is the field of view of the receiving telescope, and $B_{\text {opt }}$ is the optical bandwidth of the interference filter.

Following the method described by Bird \& Riordan (1986) an estimation of the diffuse irradiance, considering rural environment, for $830 \mathrm{~nm}$ would be between 60 and $100 \mathrm{~W} \mathrm{~m}^{-2} \mu^{-1}$, depending on the elevation angle of the Sun during the day. These values respond to the irradiance received on the ground coming from the sky in all directions without considering the solar crown, and are about the same order of the values presented in Fig. 11. Therefore, special care have to be taken from having direct sun light into the telescope field of view, situation that may produce link outages due to saturation of the photodetector.

\subsection{Probability density functions for the received optical power}

In any communication system the performance characterization is, traditionally, done by evaluating link parameter such as probability of detection, probability of miss and false alarm; threshold level for a hard-decoder and fade probability, that demands knowledge of the probability density function (PDF) for the received optical power (Wayne et al., 2010). Actually, it is rather a difficult task to determine what is the exact PDF that fits the statistics of the optical power received through an atmospheric path.

Historically, many PDF distributions have be proposed to described the random fading events of the signal-carrying optical beam, leading to power losses and eventually to complete outages. The most widely accepted distributions are the Log-Normal (LN) and the Gamma-Gamma (GG) models, although, many others have been subject of studies, namely, the $K$, Gamma, exponential, $I-K$ and Lognormal-Rician distributions (Churnside \& Frehlich, 1989; Epple, 2010; Vetelino et al., 2007).

In literature, although not always mentioned, the PDF distribution for the received optical power in a wireless link will be greatly influenced whether the receiver have a collecting aperture or it is just the bear photodetector, i.e., a point receiver. Experimental studies support the fact that the LN model is valid in weak turbulence regime for a point receiver and in all regimes of turbulence for aperture averaged data (Perlot \& Fritzsche, 2004; Vetelino et al., 2007). On the other hand, the GG model is accepted to be valid in all turbulence regimes for a point receiver, nevertheless, this does not hold when aperture averaging takes place (Al-Habash et al., 2001; Vetelino et al., 2007).

The Log-Normal distribution is given by

$$
f_{\mathrm{LN}}\left(I ; \mu_{\ln I}, \sigma_{\ln I}^{2}\right)=\frac{1}{I \sqrt{2 \pi \sigma_{\ln I}^{2}}} \exp \left\{-\frac{\left[\ln (I)-\mu_{\ln I}\right]^{2}}{2 \sigma_{\ln I}^{2}}\right\}, \quad I>0,
$$

where $\mu_{\ln I}$ is the mean and $\sigma_{\ln I}^{2}$ is the variance of the log-irradiance, and they are related to the scintillation index $\sigma_{I}^{2}$ by

$$
\begin{aligned}
& \mu_{\ln I}=\ln \langle I\rangle-\frac{\sigma_{\ln I}^{2}}{2}, \\
& \sigma_{\ln I}^{2}=\ln \left(\sigma_{I}^{2}+1\right) .
\end{aligned}
$$

The Gamma-Gamma distribution is used to model the two independent contributions of the small-scale and large-scale of turbulence, assuming each of them is governed by a Gamma 
process. The GG distribution is given by

$$
f_{\mathrm{GG}}(I ; \alpha, \beta)=\frac{2(\alpha \beta)^{(\alpha+\beta) / 2}}{\Gamma(\alpha) \Gamma(\beta)} I^{(\alpha+\beta) / 2-1} K_{\alpha-\beta}(2 \sqrt{\alpha \beta I}), \quad I>0,
$$

where $K_{n}(x)$ is the modified Bessel function of the second kind and order $n$, and, $\alpha$ and $\beta$ are parameters directly related to the effects induced by the large-scale and small-scale scattering, respectively (Epple, 2010). The parameters $\alpha$ and $\beta$ are related to the scintillation index by

$$
\sigma_{I}^{2}=\frac{1}{\alpha}+\frac{1}{\beta}+\frac{1}{\alpha \beta}
$$

It is customary to normalize Eq. (72) and Eq. (75) in the sense that $\langle I\rangle=1$. Under such assumption, the parameters $\alpha$ and $\beta$ of the GG distribution can be related to the small-scale and large-scale scintillation, introduced in Section 3.5.3, in the form (Andrews \& Philips, 2005)

$$
\begin{aligned}
& \alpha=\frac{1}{\sigma_{X}^{2}(D)}=\frac{1}{\exp \left(\sigma_{\ln X}^{2}(D)\right)-1} \\
& \beta=\frac{1}{\sigma_{Y}^{2}(D)}=\frac{1}{\exp \left(\sigma_{\ln Y}^{2}(D)\right)-1} .
\end{aligned}
$$

\section{Wireless optical communication system performance}

\subsection{Intensity-modulation direct-detection}

One of the fundamental technical decisions for a wireless optical communication systems is the choice of the modulation scheme. Although, many modulation techniques have been proposed, from non-coherent to coherent schemes, there is marked trend to favor the use of intensity-modulation direct-detection (IM/DD) scheme because it hides the high-frequency nature of the optical carrier thanks to its equivalent baseband model (Barry, 1994). Another reason to prefer the IM/DD scheme is the relatively low design complexity of the receiver system, when comparing to coherent systems, and because the photodetector is many times larger than the optical wavelength it exhibits a high degree of immunity to multipath fading (Wong et al., 2000). Among the most widespread intensity-modulation schemes for optical communications, namely on-off keying (OOK) and pulse position modulation (PPM), Chan (1982) noted that the PPM scheme is the most suitable for FSO owing to the fact that it does not rely on a threshold value to apply optimal detection.

The PPM format encodes $L$ bits of information into one symbol, or word, of duration $T_{w}$ that is divided in $M=2^{L}$ slots, by transmitting power in only one out of the $M$ possible slots. Therefore, PPM presents itself as an orthogonal modulation scheme. On the receiver side the maximum-likelihood detection is done by choosing the slot that contains the maximum count of photons-i.e. energy-over a word time, after synchronization have been achieved. The waveform for a set of bits encoded in 8-PPM before and after propagation is shown in Fig. 12 .

A communication system based on M-PPM modulation groups the input bits, at the transmitter, in blocks of length $L=\log _{2} M$, with bit rate $R_{b}$, to transmit them at a symbol rate of $R_{w}=1 / T_{w}=R_{b} / \log _{2} M$, where $T_{w}$ is the word or symbol time. Hence, the bandwidth 


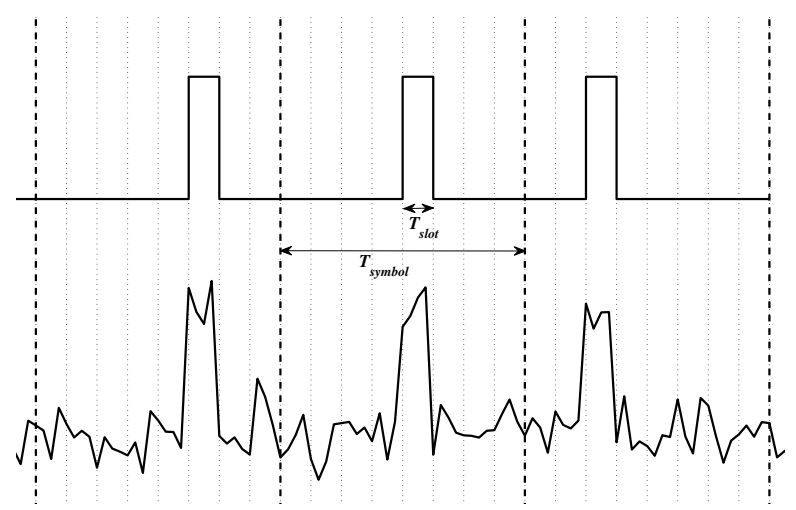

Fig. 12. Binary sequence 101100010 encoded in 8-PPM (top) and same sequence corrupted by noise (bottom).

required for transmitting with $M-P P M$ can be approximated by the bandwidth of a single slot pulse (Barry, 1994)

$$
B \approx \frac{1}{T_{p}}=\frac{1}{T_{w} / M}=\frac{M R_{b}}{\log _{2} M}
$$

where $T_{p}$ is the pulse or slot time, and corresponds to a higher requirement in bandwidth compared to that of an OOK modulation scheme, which is $B=R_{b}$. Table 4 shows the average power and bandwidth requirements for OOK and PPM modulation. The binary phase-shift keying and quadrature phase-shift keying with $N$ subcarriers modulation schemes-BPSK and N-QPSK, respectively-are presented as reference.

\begin{tabular}{ccc}
\hline \hline Modulation Scheme & Average power & Bandwidth \\
\hline OOK & $P_{\mathrm{OOK}}=\sqrt{N_{0} R_{b}} e^{-1}(B E R)$ & $R_{b}$ \\
M-PPM & $\frac{1}{\sqrt{0.5 M \log _{2} M}} P_{\mathrm{OOK}}$ & $\frac{M R_{b}}{\log _{2} M}$ \\
BPSK & $\sqrt{2} P_{\mathrm{OOK}}$ & $2 R_{b}$ \\
N-QPSK & $\sqrt{2 N} P_{\mathrm{OOK}}$ & $R_{b}$ \\
\hline \hline
\end{tabular}

Table 4. Average power and bandwidth requirements for different modulation schemes for a given bit-error rate (BER) [Adapted from Barry (1994)].

On the other hand, regarding the average optical power required for achieving a certain bit-error rate (BER), a PPM waveform need $1 / \sqrt{0.5 M \log _{2} M}$ less power than for achieving that same BER with OOK. Consequently, for a given bit rate and BER value PPM modulation demands higher bandwidth and less average optical power, when comparing with OOK. Except for the special case of 2-PPM, where the power requirement is exactly the same as for OOK, while the bandwidth is double. Furthermore, many authors have proposed new modulations derived from PPM, in particular differential PPM (DPPM) (Shiu \& Kahn, 1999), overlapping PPM (OPPM) (Patarasen \& Georghlades, 1992), improved PPM (IPPM) (Perez-Jimenez et al., 1996) and multipulse PPM (MPPM) (Sigiyama \& Nosu, 1989), aiming to overcome the excessive bandwidth requirements of PPM modulation. 
Demodulating a PPM signal can be done either by hard-decoding or soft-decoding. The latter is preferred as it requires, after slot and symbol synchronization have been achieved, to integrate the power on each slot within a frame and then choose the largest. This way the PPM is highly resistance to background noise and the receiver sensibility is increased respect to the hard-decoding approach, where a simple threshold detector is used to decide if a "low" or "high" have been received.

As the information is conveyed in time for PPM modulation format, a critical issue is the synchronization procedure. Timing recovery of the slot and symbol clock become essential to correctly decode the received noisy waveform. Many strategies have been proposed to aid in the synchronization stage (Georghiades \& Snyder, 1984; Srinivasan et al., 2005; Sun \& Davidson, 1990). Moreover, variants of PPM have been introduced, such as half-pulse PPM (Otte et al., 1998) and digital pulse interval modulation (DPIM) (Okazaki, 1978), in order to simplify the synchronization process.

\subsection{Signal-to-noise ratio in an APD photodetector}

Several terms of noise have to be taken into account for the evaluation of the signal-to-noise ratio (SNR) in an optical link. Some of them are characteristic of the photo-detector device, as the noise associated to the dark current or the noise coming from the intrinsic gain physical mechanism. Other terms come from the amplifier electronics.

The usual expression for the SNR at the receiver output is

$$
\mathrm{SNR}=\frac{i_{S}^{2}}{\sigma_{S}^{2}+\sigma_{B}^{2}+\sigma_{D}^{2}+\sigma_{A}^{2}+i_{n}^{2}}
$$

being $i_{S}^{2}$ the generated photocurrent, $\sigma_{S}^{2}$ the shot noise associated to the received signal, $\sigma_{D}^{2}$ the dark current noise, $\sigma_{B}^{2}$ the noise coming from the background optical power, $i_{n}^{2}$ the thermal noise and $\sigma_{A}^{2}$ the total equivalent noise input current associated to the amplifier. The photocurrent is calculated as follows

$$
i_{S}=\eta e G \frac{\lambda P_{S}}{h c}
$$

where $\eta$ is the quantum efficiency, $P_{S}$ is the received signal optical power, $h$ is the Planck's constant, $e$ is the electron charge, $c$ is the speed of light in vacuum and $G$ is the photo-detector intrinsic gain. The photocurrent can also be expressed by

$$
i_{S}=R_{I} P_{S},
$$

where $R_{I}$ is the current responsivity,

$$
R_{I}=\eta e G \frac{\lambda}{h c} \quad\left[\frac{\mathrm{A}}{\mathrm{W}}\right] .
$$

\subsubsection{Noise sources}

To complete the characterization of the signal-to-noise ratio an analysis of the noise sources in an avalanche photodiode (APD) have to be done. Some noise terms depend directly on 
the photodetector physical characteristics, while others are generated by the optical power illuminating the surface of the APD.

The process that detects the optical power impinging the detector is described by the occurrence of independent random events, modeled by the Poisson distribution, as an optical wave is ultimately formed by photons carrying quantized amounts of energy. This randomness in the detection process of any photon illumination the APD, is what gives rise to the shot noise and background noise. The shot noise is given by

$$
\sigma_{S}^{2}=2 e i_{S} B G F=2 e\left(R_{I} P_{S}\right) B G F,
$$

and the background noise by

$$
\sigma_{B}^{2}=2 e i_{B} B G F=2 e\left(R_{I} P_{B}\right) B G F,
$$

where $B$ is the electrical bandwidth, $P_{B}$ is the background optical power, and $F$ the excess noise factor. For a positive-intrinsic-negative (PIN) photodiode this excess noise factor is the unity, as no internal gain exists. For an APD the general expression is

$$
F=k_{e f f} G+\left(1-k_{e f f}\right)\left(2-\frac{1}{G}\right)
$$

where $k_{\text {eff }}$ is the carrier ionization ratio, for which 0.01-0.1 is the typical range of values.

Every photodetector, whether is a PIN diode or an APD, generates a drift current even when no photons are entering the detector surface. This phenomena is due to the random generation of electron-holes pairs within the depletion area, and charges are attracted by the electric field produced by polarization voltage. The dark current is the result of two current terms, and it is defined by

$$
i_{D}=i_{D S}+i_{D B} G,
$$

where $i_{D S}$ is the surface leakage current, and $i_{D B}$ the bulk noise current (for gain unity). The second term is a function of the gain, as it is affected by the avalanche process. Then, the corresponding noise term is

$$
\sigma_{D}^{2}=2 e\left(i_{D S}+i_{D B} G^{2} F\right) B
$$

In an actual system the photodetector is always followed by an amplifier in order to adequate the signal to next stages, in the receiver chain. The amplifier noise is characterized by means of two noisy sources at the input, namely, an equivalent noise voltage source and an equivalent noise current source. The values of these sources appears as two parameters of the amplifier in the datasheet provided by the manufacturer, most of times even with plots showing their behaviour as a function of the modulated signal frequency. In a first approximation the total equivalent noise current of the amplifier can be written as

$$
\sigma_{A}^{2}=\left(i_{n A}^{2}+\frac{e_{n A}^{2}}{R^{2}}\right) B,
$$

being $R$ the feedback resistor in the transimpedance amplifier scheme, $i_{n A}$ the amplifier equivalent noise current density, in $\mathrm{A} / \sqrt{\mathrm{Hz}}$, and $e_{n A}$ the amplifier equivalent noise voltage density, in $\mathrm{V} / \sqrt{\mathrm{Hz}}$. 
Finally, as in any electronic system there will always exist thermal noise, defined by

$$
i_{n R}^{2}=\frac{4 K T B}{R},
$$

where $K$ is the Boltzmann's constant and $T$ is the absolute temperature in Kelvins.

Traditionally the manufacturer gives a characteristic noise figure of the photo-detector known as noise equivalent power (NEP). This is defined as the minimum optical signal that could be detected by the device, where minimum signal is understood as the signal power level for which the SNR equals unity. For a bared APD the NEP is limited by the dark current, whereas for a complete photodetector module other terms must be included, namely, the electronic and the thermal noise. Moreover, it is a common practice to give that parameter for $1 \mathrm{~Hz}$ bandwidth, i.e., normalized with respect to the bandwidth, which may vary from one application to other. The NEP for a complete photodetector (APD plus preamplifier) is given by

$$
\mathrm{NEP}=\left.\frac{P_{S}}{\sqrt{B}}\right|_{\mathrm{SNR}=1}=\frac{1}{R_{I}} \sqrt{\frac{1}{B}\left(\sigma_{D}^{2}+\sigma_{A}^{2}+i_{n}^{2}\right)} \quad\left[\frac{\mathrm{W}}{\sqrt{\mathrm{Hz}}}\right] .
$$

By using this merit figure the evaluation of the noise could be abbreviated, as there is no need to calculate all of the terms of noise involved in it. Therefore, the SNR calculation only implies the knowledge of the signal and background power, along with the photodetector NEP, thus

$$
\mathrm{SNR}=\frac{i_{s}^{2}}{\sigma_{S}^{2}+\sigma_{B}^{2}+R_{I}^{2} \mathrm{NEP}^{2} B} .
$$

\subsection{Bit-error rate performance}

In digital communication systems, reliability is commonly expressed as the probability of bit error, best known as bit-error rate (BER), measured at the output of the receiver and depends directly on the received signal level and receiver noise level. The smaller the BER, the more reliable the communication system .

In order to obtain an accurate calculation of the BER, it is necessary to know the probability density function of the receiver output signal. In the case of WOC systems the APD is the preferred choice as photodetector. The output of an APD is modeled by the McIntyre-Conradi distribution (Webb et al., 1974), although, the Gaussian approximation is sufficient enough when the bulk current is of the order of nanoampere and the absorbed photons are more than a few hundred within the observation time (Davidson \& Sun, 1988; Ricklin et al., 2004).

For the output of a wireless optical communication link, with an APD as photodetector, there are two possibilities, namely, that a pulse is transmitted or not. In the former situation the APD is detecting optical power corresponding to the signal level and the background radiation, while in the latter only background radiation is received. Assuming a Gaussian distribution given by

$$
f\left(x ; \mu, \sigma^{2}\right)=\frac{1}{\sqrt{2 \pi \sigma^{2}}} \exp \left[-\frac{(x-\mu)^{2}}{2 \sigma^{2}}\right],
$$


the average current $\mu_{1}$ and its associated current noise $\sigma_{1}^{2}$ generated at the APD's output when a pulse have been transmitted is

$$
\begin{aligned}
& \mu_{1}=e G \frac{\eta}{h v}\left(P_{S}+P_{B G}\right)+i_{D S}+i_{D B} G \\
& \sigma_{1}^{2}=2 B\left(\frac{e^{2} \eta}{h v} F G^{2}\left(P_{S}+P_{B G}\right)+\frac{2 K T}{R_{L}}+e\left(i_{D S}+i_{D B} G^{2} F\right)\right) .
\end{aligned}
$$

On the other hand, when no pulse is transmitted the average current $\mu_{0}$ and its associated current noise $\sigma_{0}^{2}$ is

$$
\begin{aligned}
& \mu_{0}=e G \frac{\eta}{h v}\left(\epsilon P_{S}+P_{B G}\right)+i_{D S}+i_{D B} G \\
& \sigma_{0}^{2}=2 B\left(\frac{e^{2} \eta}{h v} F G^{2}\left(\epsilon P_{S}+P_{B G}\right)+\frac{2 K T}{R_{L}}+e\left(i_{D S}+i_{D B} G^{2} F\right)\right),
\end{aligned}
$$

where $\epsilon$ denotes the laser extinction ratio, generating residual light even when no pulse is being transmitted. All other parameters in Eq. (94) to Eq. (97) were presented in Section 6.2.1.

\subsubsection{Probability of error for on-off keying modulation}

The simplest signaling format in a digital wireless optical communication system is the on-off keying $(\mathrm{OOK})$, where a binary ' 1 ' is represented by a pulse while a binary ' 0 ' is represented by the absence of a pulse. The receiver, in this case, is comprised of a threshold detector for deciding which symbol have been received. Assuming that the receiver output noise follows a white Gaussian model, the corresponding PDFs for the cases of a pulse and no pulse being transmitted are shown in Fig. 13, where $\tau$ represents the threshold level applied for comparison, and, the mean and variances are defined in Eq. (94) to Eq. (97). Let us consider

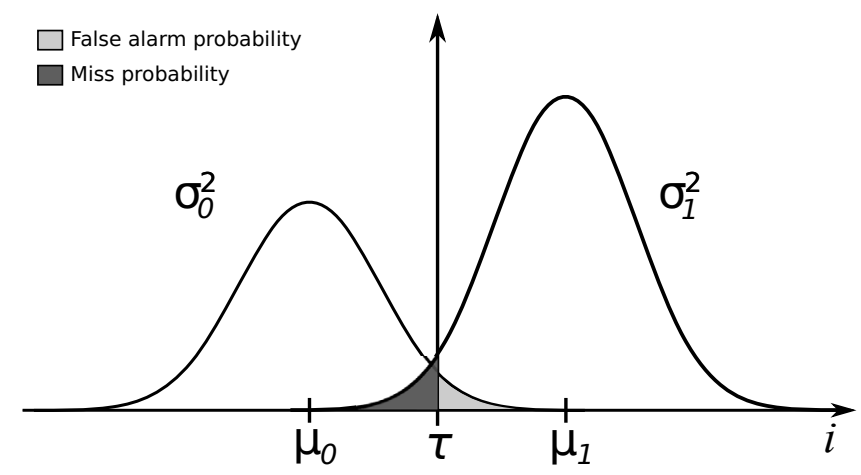

Fig. 13. Received signal p.d.f. under white Gaussian noise assumption.

now the word-error probability (PWE), which is compound of two types of errors. If the received signal level is higher than the set threshold $\tau$ when no pulse has been transmitted by the source, a false alarm is generated. On the contrary, if a pulse indeed has been transmitted and the received signal level is lower than $\tau$, then, a miss is produced. Thus, the PWE is given 
by

$$
\begin{aligned}
\mathrm{PWE} & =P_{\mathrm{FA}} P_{0}+P_{\mathrm{Miss}} P_{1}, \\
P_{\mathrm{FA}} & =Q\left(\frac{\tau-\mu_{0}}{\sigma_{0}}\right), \\
P_{\mathrm{Miss}} & =1-Q\left(\frac{\tau-\mu_{1}}{\sigma_{1}}\right)=Q\left(\frac{\mu_{1}-\tau}{\sigma_{1}}\right),
\end{aligned}
$$

where $P_{\mathrm{FA}}$ denotes the probability of false alarm, $P_{\text {Miss }}$ is the probability of miss, and $Q(x)$ is the Gaussian Q-function defined as

$$
Q(x)=\frac{1}{2 \pi} \int_{x}^{\infty} e^{-x^{2} / 2} d x .
$$

Whenever an equiprobable signaling system is used, the probability of receiving a pulse or not are equal, this is $P_{0}=P_{1}=\frac{1}{2}$. For OOK modulation, the bit-error probability $P_{b}$ is the same as the word-error probability-i.e. $P_{b}=$ PWE.

The problem of defining the optimum threshold level have been addressed before, and the expression for $\tau$ in a maximum-likelihood receiver yields to (Ricklin et al., 2004)

$$
\left(\frac{\sigma_{1}^{2}}{\sigma_{0}^{2}}-1\right) \tau^{2}+2\left(\mu_{1}-\frac{\sigma_{1}^{2}}{\sigma_{0}^{2}} \mu_{0}\right) \tau-\sigma_{1}^{2} \ln \left(\frac{\sigma_{1}^{2}}{\sigma_{0}^{2}}\right)+\frac{\sigma_{1}^{2}}{\sigma_{0}^{2}} \mu_{0}^{2}-\mu_{1}^{2}=0 .
$$

Nevertheless, as real-time calculation of the mean and variance of the received signal is rather a complex task, a reasonable approach is to set the threshold level to half of the signal amplitude, which actually approaches the optimum value of $\tau$ for high SNR values.

\subsubsection{Probability of error for M-ary pulse position modulation}

Pulse position modulation is a signaling format well suited for laser applications, requiring low average power and is very resistant to background radiation. In M-ary PPM signaling, $L$ binary source bits are transmitted as a single light pulse in one out of $M=2^{L}$ possible time slots, once every $T_{w}$ seconds.

A maximum-likelihood APD based receiver, with M-PPM modulation, have a word-error probability given by (Dolinar et al., 2006)

$$
\mathrm{PWE}=1-\int_{-\infty}^{\infty} \sqrt{\frac{\gamma}{\beta+\gamma}} \phi\left(\sqrt{\frac{\gamma}{\beta+\gamma}}(x-\sqrt{\beta})\right) \Phi(x)^{M-1} d x,
$$

where $\phi(x)$ is given by Eq. (93) with zero mean and unitary variance, $\Phi(x)$ is the standard Gaussian cumulative distribution function, $\beta=\left(\mu_{1}-\mu_{0}\right)^{2} / \sigma_{0}^{2}$ is the symbol signal-to-noise ratio, and, $\left.\gamma=\left(\mu_{1}-\mu_{0}\right)^{2}\right) /\left(\sigma_{1}^{2}-\sigma_{0}^{2}\right)$.

In a different approach, a threshold detector can be implemented for demodulating PPM signals. Although, it is not the optimum strategy it can greatly simplify receiver design, as tight synchronization requirements have not to be pursued as for the optimum receiver. 
The expression for the word-error probability for a threshold receiver have been derive by Moreira et al. (1996), leading to

$$
\mathrm{PWE}=1-\left[P_{1}+\frac{1}{M} P_{2}+\sum_{n=2}^{M} \frac{1}{n} P_{3 n}\right],
$$

where $P_{1}$ is the probability of detecting a pulse in the correct position, $P_{2}$ is the probability of that no pulse is detected and $P_{3 n}$ is the probability of detecting $n$ pulses. These probabilities are defined by

$$
\begin{aligned}
P_{1} & =\left(1-P_{\mathrm{Miss}}\right)\left(1-P_{\mathrm{FA}}\right)^{M-1}, \\
P_{2} & =P_{\mathrm{Miss}}\left(1-P_{\mathrm{Miss}}\right)^{M-1}, \\
P_{3 n} & =\left(\begin{array}{c}
M-1 \\
n-1
\end{array}\right)\left(1-P_{\mathrm{Miss}}\right) P_{\mathrm{FA}}^{n-1}\left(1-P_{\mathrm{FA}}\right)^{M-1},
\end{aligned}
$$

where $P_{\mathrm{FA}}$ and $P_{\text {Miss }}$ are given by Eq. (99) and Eq. (100), respectively.

Sometimes having the error probability at bit level is desirable. Thus, for a M-ary orthogonal signaling system, the probability of word error can be converted to bit-error probability according to (Proakis, 2001)

$$
P_{b}=\frac{M / 2}{M-1} \mathrm{PWE}
$$

\subsection{Bit-error rate under turbulent atmosphere}

In the presence of optical turbulence, the probability of error is a conditional probability owing to the random nature of the received optical power. Thus, the SNR becomes a random variable and consequently the PWE have to be averaged over all the possible received optical signal levels, according to the proper statistical distribution model of the irradiance $I$. This yields to

$$
\operatorname{PWE}\left(\sigma_{I}^{2}\right)=\int_{0}^{\infty} P(I) P W E(I) d I, \quad I>0,
$$

where $\sigma_{I}^{2}$ is the scintillation index defined in Section 3, which depends directly on link's parameters such as $C_{n}^{2}$, link distance, laser divergence, and aperture averaging among others.

As stated in Section 5.3 the Log-Normal model is the most accepted in weak turbulence regimes for point receivers, and in all regimes of turbulence when aperture averaging takes place. Under weak turbulence regime the scintillation index, given by Eq. (52), can be expressed as

$$
\sigma_{I}^{2} \cong \sigma_{\ln I}^{2}
$$

and normalizing the irradiance in the sense that $\langle I\rangle=1$, Eq. (72) reduces to

$$
P(I)=\frac{1}{I \sqrt{2 \pi \sigma_{I}^{2}}} \exp \left[-\frac{\left(\ln I+\frac{\sigma_{I}^{2}}{2}\right)^{2}}{2 \sigma_{I}^{2}}\right] .
$$

Some examples of the performance of a wireless optical communication system, with 

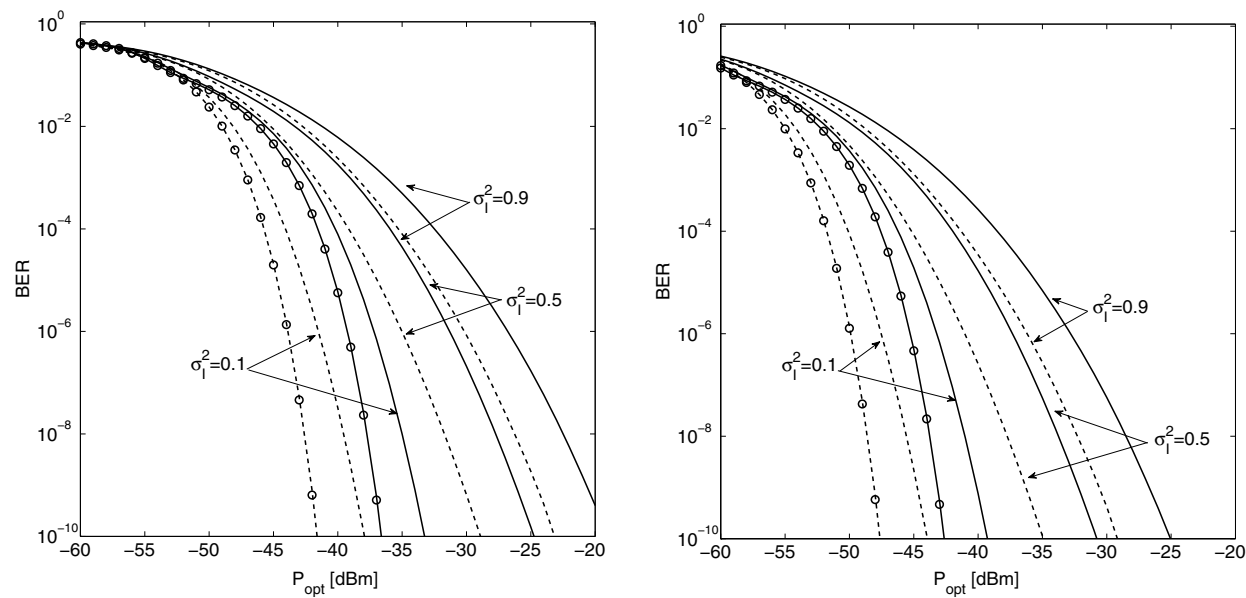

(a) Performance of OOK modulation for fixed (b) Performance of 8-PPM modulation for fixed threshold (solid line) and optimum threshold threshold (solid line) and optimum threshold (dashed lines) receivers under different turbulence (dashed lines) receivers under different turbulence levels. Lines with circles represent free-space levels. Lines with circles represent free-space (no-turbulence) conditions. (no-turbulence) conditions.

Fig. 14. Performance of OOK and 8-PPM modulation for bit-error rate vs received average optical power in a WOC systems, with IM/DD, for different levels of scintillation index.

intensity-modulation and direct-detection, for OOK and PPM modulation with fixed and optimum threshold are shown in Fig. 14(a) and Fig. 14(b), respectively, for different levels of scintillation index. It should be noted that the SI includes all the parameters such as laser wavelength and beam divergence, aperture averaging, the refractive-index structure parameter, link distance, transmitting and receiving aperture diameters, among others. It is readily seen, from Fig. 14, the tremendous impact of atmospheric turbulence in wireless optical communication systems regardless the modulation scheme being used, although, a PPM system in general performs better than OOK respect to the average optical power needed for achieving a desired bit-error rate level. For example, both for OOK and 8-PPM, there is roughly a $7 \mathrm{~dB}$ power penalty for a $\mathrm{BER}=10^{-6}$ with fixed threshold receiver (solid lines) and $\sigma_{I}^{2}=0.5$ respect to the case of no turbulence, i.e. free-space conditions.

For a more comprehensive analysis of a pulse position modulation receiver Fig. 15 is presented. The performance of PPM maximum-likelihood receiver with modulation orders up to 16 is shown in Fig. 15(a), where it becomes evident that increasing the PPM modulation order to the next permitted one there is an improvement of $3 \mathrm{~dB}$ respect to the average optical power needed to achieve a certain BER. Figure 15(b) is presented as a mean of comparison between the performance of a maximum-likelihood receiver and an optimum threshold receiver for PPM modulation. The penalty incurred for using the optimum threshold receiver instead of the maximum-likelihood receiver is about $1 \mathrm{~dB}$ for BER $<10^{-4}$. Nevertheless, this penalty increases for higher values of bit-error rate, although, a typical communication system with no forward error correction (FEC) code implemented will be designed to have a BER lower than $10^{-4}$. 

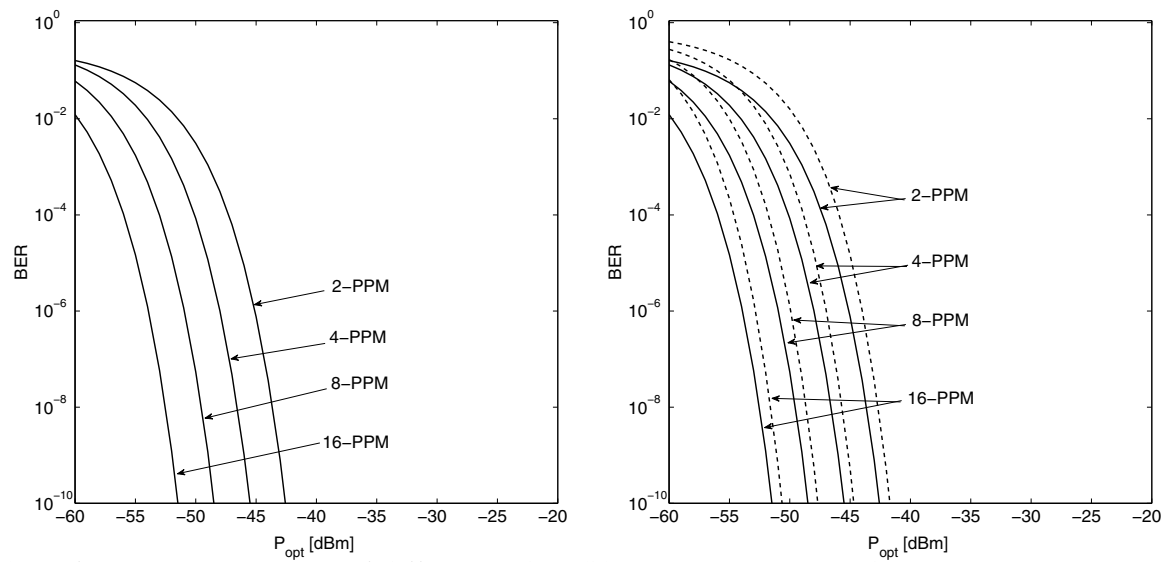

(a) Performance comparison of different orders (b) Comparison between a PPM of PPM modulation with maximum-likelihood optimum threshold receiver and a PPM receiver. maximum-likelihood receiver (solid line).

Fig. 15. Performance of a PPM optimal threshold receiver and PPM maximum-likelihood receiver vs received average optical power under free-space (no-turbulence) conditions.

For the calculations in Fig. 14 and Fig. 15 background radiation was omitted in the analysis and it was assumed an APD's current gain $G=70, k_{e f f}=0.094$, quantum efficiency $\eta=0.77$ and NEP $=55 \mathrm{fW} / \sqrt{\mathrm{Hz}}$. The light source was set to $\lambda=780 \mathrm{~nm}$, pulse time $T_{p}=1 \mathrm{~ns}$ and extinction ratio $\epsilon=0.001$.

\section{References}

Al-Habash, M. A., Andrews, L. C. \& Philips, R. L. (2001). Mathematical model for the irradiance probability density function of a laser beam propagating through turbulent media, Opt. Eng. 40(8): 1554-1562.

Andreas, E. L. (1988). Estimating $c_{n}^{2}$ over snow and sea ice from meteorological data, J. Opt. Soc. Am. A 5(4): 481-495.

Andrews, L. C. (1992). Aperture-averaging factor for optical scintillations of plane and spherical waves in the atmosphere, J. Opt. Soc. Am. 9(4): 597-600.

Andrews, L. C., Al-Habash, M. A., Hopen, C. Y. \& Phillips, R. L. (2001). Theory of optical scintillation: Gaussian-beam wave model, Waves in Random and Complex Media 11(3): 271-291.

Andrews, L. C. \& Philips, R. L. (2005). Laser Beam Propagation through Random Media, 2nd edn, SPIE Press, Belligham.

Arimoto, Y. (2010). Near field laser transmission with bidirectional beacon tracking for tbps class wireless communications, in H. Hemmati (ed.), Free-Space Laser Communication Technologies XXII, Vol. 7587 of Proc. SPIE, pp. 758708-1-758708-8.

Barry, J. (1994). Wirelees Infrarred Communications, The Kluwer International Series in Engineering and Computer Science, Kluwer Academic Publishers, Norwell. 
Bird, R. E. \& Riordan, C. (1986). Simple solar spectral model for direct and diffuse irradiance on horizontal and tilted plans at the earth's surface for cloudless atmosphere, J. Clim. Meteor. 25: 87-97.

Cazaubiel, V., Troy, B. \& Chene, B. (2006). Optical DATA relay satellite architecture and airborne laser communication demonstrator, Emerging and Future Technologies for Space Based Operations Support to NATO Military Operations, Proc. RTO-MP-RTB-SPSM-001, pp. 20-1-20-10.

Chan, V. (1982). Coding for the turbulent atmospheric optical channel, IEEE Trans. Commun. 30(1): 269-275.

Churnside, J. H. (1991). Aperture averaging of optical scintillations in the turbulent atmosphere, Appl. Opt. 30: 1982-1994.

Churnside, J. H. \& Frehlich, R. G. (1989). Experimental evaluation of log-normally modulated rician and ik models of optical scintillation in the atmosphere, J. Opt. Soc. Am. A 6(11): 1760-1766.

Churnside, J. H. \& Lataitis, R. J. (1990). Wander of an optical beam in the turbulent atmosphere, Appl. Opt. 29(7): 926-930.

Ciaramella, E., Arimoto, Y., Contestabile, G., Presi, M., D’Errico, A., Guarino, E. \& Matsumoto, M. (2009). 1.28 Terabit/s (32x40 Gbit/s) WDM transmission system for free space optical communications, IEEE J. Sel. Area Comm. 27: 1639-1645.

Davidson, F. M. \& Sun, X. (1988). Gaussian approximation versus nearly exact performance analysis of optical communication systems with ppm signaling and apd receivers, IEEE. Trans. Commun. 36(11): 1185-1192.

Dios, F., Recolons, J., Rodríguez, A. \& Batet, O. (2008). Temporal analysis of laser beam propagation in the atmosphere using computer-generated long phase screens, Opt. Express 16(3): 2206-2220.

Dolinar, S. J., Hamkins, J., Moision, B. E. \& Vilnrotter, V. A. (2006). Deep Space Optical Communications, Wiley-Interscience, chapter Optical Modulation and Coding, pp. 215-299.

Doss-Hammel, S., Oh, E., Ricklin, J., Eaton, F., Gilbreath, C. \& Tsintikidis, D. (2004). A comparison of optical turbulence models, in J. C. Ricklin \& D. G. Voelz (eds), Remote Sensing and Modeling of Ecosystems for Sustainability, Vol. 5550 of Proc. SPIE, pp. 236-246.

Epple, B. (2010). Simplified channel model for simulation of free-space optical communications, J. Opt. Commun. Netw. 2(5): 293-304.

Fante, R. L. (1980). Electromagnetic beam propagation in turbulent media: an update, Proc. IEEE 68(11): 1424-1443.

Fleck, J. A., Morris, J. R. \& Feit, M. D. (1976). Time-dependent propagation of high-energy laser beams through the atmosphere, Appl. Phys. 10: 129-160.

Fletcher, G., Hicks, T. \& Laurent, B. (1991). The SILEX optical interorbit link experiment, J. Electron. Comm. Eng. 3(6): 273-279.

Frehlich, R. (2000). Simulation of laser propagation in a turbulent atmosphere, J. Opt. Soc. Am. 39(3): 393-397.

Fried, D. L. (1967). Aperture averaging of scintillation, J. Opt. Soc. Am. 57(2): 169-175.

Georghiades, C. N. \& Snyder, D. L. (1984). Locating data frames in direct-detection optical communication systems, IEEE Trans. Commun. COM-32(2): 118-123.

Harding, C. M., Johnston, R. A. \& Lane, R. G. (1999). Fast simulation of a kolmogorov phase screen, Appl. Opt. 38(11): 2161-2160. 
Hudson, R. D. (1969). Infrared System Engineering, John Wiley \& Sons, New York.

Ishimaru, A. (1969). Fluctuations of a focused beam wave for atmospheric turbulence probing, Proc. IEEE 57: 407-414.

Jeganathan, M., Toyoshima, M., Wilson, K. ., James, J., Xu, G. \& Lesh, J. (1997). Data analysis results from the GOLD experiments, Free-Space Laser Comm. Tech. IX, Vol. 1990 of Proc. SPIE, pp. 70-81.

Kim, I. I., Stieger, R., Koontz, J. A., Moursund, C., Barclay, M., Adhikari, M., Schuster, J., Korevaar, E., Ruigrok, R. \& DeCusatis, C. (1998). Wireless optical transmission of fast ethernet, FDDI, ATM, and ESCON protocol data using the TerraLink laser communication system, Opt. Eng. 37(12): 3143-3155.

Knestrick, G. L. \& Curcio, J. A. (1967). Measurements of spectral radiance of the horizon sky, Appl. Opt. 6(12): 2105-2109.

Kolmogorov, A. N. (1941). The local structure of turbulence in a incompressible viscous fluid for very large reynolds numbers, C. R. (Doki) Acad. Sci. U.S.R.R. 30: 301-305.

Lane, R. G., Glindemann, A. \& Dainty, J. C. (1992). Simulation of a kolmogorov phase screen, Waves Random Media 2: 209-224.

Lawrence, R. S., Ochs, G. R. \& Clifford, S. F. (1970). Measurements of atmospheric turbulence relevant to optical propagation, J. Opt. Soc. Am. 60(6): 826-830.

Levine, B., Martinsen, E., Wirth, A., Jankevics, A., Toledo-Quinones, M., Landers, F. \& Bruno, T. (1998). Horizontal line-of-sight turbulence over near-ground paths and implications for adaptive optics corrections in laser communications, Appl. Opt. 37(21): 4782-4788.

Martin, J. M. \& Flattè, S. M. (1998). Intensity images and statistics from numerical simulation of wave propagation in 3-d random media, J. Opt. Soc. Am. 27(11): 2111-2126.

Moreira, A. J. C., Valdas, R. T. \& de Oliveira Duarte, A. M. (1996). Performance of infrared transmission systems under ambient light interference, IEE Proc. Optoelectron. 143(6): 339-346.

Ochs, G. R. \& Hill, R. J. (1985). Optical-scintillation method of measuring turbulence inner scale, Appl. Opt. 24(15): 2430-2432.

Okazaki, A. (1978). Pulse interval modulation applicable to narrow band transmission, IEEE. Trans. on Cable Television CATV-3(4): 155-164.

Otte, R., de Jong, L. P. \& van Roermund, A. H. M. (1998). Slot synchronization by reducing the ppm pulsewidth in wireless optical systems, IEEE Trans. on Circuits and Systems 45(7): 901-903.

Patarasen, S. \& Georghlades, C. N. (1992). Frame synchronization for optical overlapping pulse-position modulation systems, IEEE Trans. Commun. 40(4): 783-794.

Perez-Jimenez, R., Rabadan, J., Melian, V. \& Betancor, M. (1996). Improved ppm modulations for high spectral efficiency ir-wlan, Personal, Indoor and Mobile Radio Communications, 1996. PIMRC'96., Seventh IEEE International Symposium on, Vol. 1, pp. 262-266.

Perlot, N. \& Fritzsche, D. (2004). Aperture-averaging - theory and measurements, in G. S. Mecherle, C. Y. Young \& J. S. Stryjewski (eds), Free-Space Laser Communication Technologies XVI, Vol. 5338 of Proc. SPIE, pp. 233-242.

Proakis, J. G. (2001). Digital Communications, McGraw-Hill, Boston.

Recolons, J., Andrews, L. \& Philips, R. L. (2007). Analysis of beam wander effects for a horizontal-path propagating gaussian-beam wave: focused beam case, Opt. Eng. 46(8): 086002-1-086002-11. 
Recolons, J. \& Dios, F. (2005). Accurate calculation of phase screens for the modelling of laser beam propagation through atmospheric turbulence, in S. M. Doss-Hammel \& A. Kohnle (eds), Atmospheric Optical Modeling, Measurement, and Simulation, Vol. 5891 of Proc. SPIE, pp. 51-62.

Ricklin, J. C., Bucaille, S. \& Davidson, F. M. (2004). Performance loss factors for optical communication through clear air turbulence, in D. G. Voelz \& J. C. Ricklin (eds), Free-Space Laser Communications and Active Laser Illumination III, Vol. 5160 of Proc. SPIE, pp. 1-12.

Ricklin, J. C., Hammel, S. M., Eaton, F. D. \& Lachinova, S. L. (2006). Atmospheric channel effects on free-space laser communication, J. Opt. Fiber. Commun. Rep. 3: 111-158.

Sadot, D. \& Kopeika, N. S. (1992). Forecasting optical turbulence strength on the basis of macroscale meteorology and aerosols: models and validation, Opt. Eng. 31(31): 200-212.

Shiu, D. \& Kahn, J. M. (1999). Differential pulse-position modulation for power-efficient optical communication, IEEE Trans. on Commun. 47(8): 1201-1211.

Sigiyama, H. \& Nosu, K. (1989). MPPM: A method for improving the band-utilization efficiency in optical PPM, IEEE J. Lightwave Techn. 7(3): 465-472.

Sova, R. M., Sluz, J. E., Young, D. W., Juarez, J. C., Dwivedi, A., Demidovich, I. N. M., Graves, J. E., Northcott, M., Douglass, J., Phillips, J., Driver, D., McClarin, A. \& Abelson, D. (2006). $80 \mathrm{gb} / \mathrm{s}$ free-space optical communication demonstration between an aerostat and a ground station, in A. K. Majumdar \& C. C. Davis (eds), Free-Space Laser Communications VI, Vol. 6304 of Proc. SPIE, p. 630414.

Srinivasan, M., Vilnrotter, V. \& Lee, C. (2005). Decision-directed slot synchronization for pulse-position-modulated optical signals, The Interplanetary Network Progress Report 42-161, Jet Propulsion Laboratory, Pasadena, California.

Sun, X. \& Davidson, F. M. (1990). Word timing recovery in direct detection optical ppm communication systems with avalanche photodiodes using a phase lock loop, IEEE Trans. Commun. 38(5): 666-673.

Tatarskii, V. I. (1971). The effects of the turbulent atmosphere on wave propagation, Israel Program for Scientific Translation, Jerusalem.

Tyson, R. K. (2002). Bit-error rate for free-space adaptive optics laser communications, J. Opt. Soc. Am. A 19(4): 753-758.

Vetelino, F. S., Young, C. \& Andrews, L. (2007). Fade statistics and aperture averaging for gaussian beam waves in moderate-to-strong turbulence, Appl. Opt. 46(18): 3780-3790.

Wang, T., Ochs, G. R. \& Clifford, S. F. (1978). A saturation-resistant optical scintillometer to measure $C_{n}^{2}$, J. Opt. Soc. Am. 68(3): 334-338.

Wayne, D. T., Philips, R. L. \& Andrews, L. C. (2010). Comparing the log-normal and gamma-gamma model to experimental probability density functions of aperture averaging data, in C. Majumdar, A. K.and Davis (ed.), Free-Space Laser Communications X, Vol. 7814 of Proc. SPIE, p. 78140K.

Webb, P. P., McIntyre, R. J. \& Conradi, J. (1974). Properties of avalanche photodiodes, RCA review 35: 234-278.

Weyrauch, T. \& Vorontsov, M. A. (2004). Free-space laser communications with adaptive optics: Atmospheric compensation experiments, J. Opt. Fiber. Commun. Rep. 1: 355-379. 
Wong, K. K., R, T. O. \& Kiatweerasakul, M. (2000). The performance of optical wireless ook, 2-ppm and spread spectrum under the effects of multipath dispersion and artificial light interference, Int. J. Commun. Syst. 13: 551-576.

Yitzhaky, Y., Dror, I. \& Kopeika, N. S. (1997). Restoration of atmospherically blurred images according to weather-predicted atmospheric modulation transfer functions, Opt. Eng. 36(11): 3064-3072.

Yura, H. T., Sung, C. C., Clifford, S. F. \& Hill, R. J. (1983). Second-order rytov approximation, J. Opt. Soc. Am. 73(4): 500-502. 


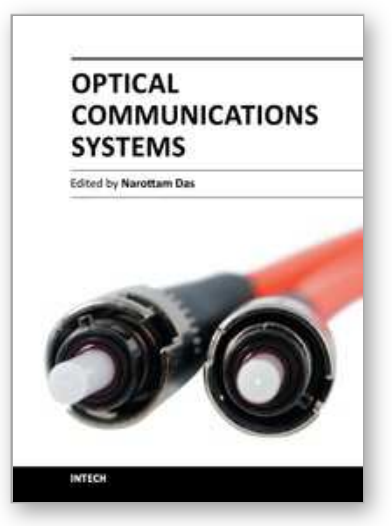

\author{
Optical Communications Systems \\ Edited by Dr. Narottam Das
}

ISBN 978-953-51-0170-3

Hard cover, 262 pages

Publisher InTech

Published online 07, March, 2012

Published in print edition March, 2012

Optical communications systems are very important for all types of telecommunications and networks. They consists of a transmitter that encodes a message into an optical signal, a channel that carries the signal to its destination, and a receiver that reproduces the message from the received optical signal. This book presents up to date results on communication systems, along with the explanations of their relevance, from leading researchers in this field. Its chapters cover general concepts of optical and wireless optical communication systems, optical amplifiers and networks, optical multiplexing and demultiplexing for optical communication systems, and network traffic engineering. Recently, wavelength conversion and other enhanced signal processing functions are also considered in depth for optical communications systems. The researcher has also concentrated on wavelength conversion, switching, demultiplexing in the time domain and other enhanced functions for optical communications systems. This book is targeted at research, development and design engineers from the teams in manufacturing industry; academia and telecommunications service operators/ providers.

\title{
How to reference
}

In order to correctly reference this scholarly work, feel free to copy and paste the following:

Ricardo Barrios and Federico Dios (2012). Wireless Optical Communications Through the Turbulent Atmosphere: A Review, Optical Communications Systems, Dr. Narottam Das (Ed.), ISBN: 978-953-51-0170-3, InTech, Available from: http://www.intechopen.com/books/optical-communications-systems/wireless-opticalcommunications-through-the-turbulent-atmosphere-a-review

\section{INTECH}

open science | open minds

\section{InTech Europe}

University Campus STeP Ri

Slavka Krautzeka 83/A

51000 Rijeka, Croatia

Phone: +385 (51) 770447

Fax: +385 (51) 686166

www.intechopen.com

\section{InTech China}

Unit 405, Office Block, Hotel Equatorial Shanghai

No.65, Yan An Road (West), Shanghai, 200040, China 中国上海市延安西路65号上海国际贵都大饭店办公楼 405 单元

Phone: +86-21-62489820

Fax: $+86-21-62489821$ 